\title{
A multivariate spatial mixture model for areal data: examining regional differences in standardized test scores
}

\author{
Brian Neelon and Alan E. Gelfand \\ Duke University, Durham, USA \\ and Marie Lynn Miranda \\ University of Michigan, Ann Arbor, USA
}

[Received March 2012. Final revision November 2013]

\begin{abstract}
Summary. Motivated by a study exploring geographic disparities in test scores among fourth graders in North Carolina, we develop a multivariate mixture model for the spatial analysis of correlated continuous outcomes. The responses are modelled as a finite mixture of multivariate normal distributions, which accommodates a wide range of marginal response distributions and allows investigators to examine covariate effects within subpopulations of interest. The model has a hierarchical structure incorporating both individual and areal level predictors as well as spatial random effects for each mixture component. Conditional auto-regressive priors on the random effects provide spatial smoothing and allow the shape of the multivariate distribution to vary flexibly across geographic regions. By integrating over this distribution, we obtain regionspecific joint, marginal and conditional inferences of interest. We adopt a Bayesian modelling approach and develop an efficient posterior sampling algorithm that relies primarily on closed form full conditionals. Our results show that students in the central and coastal counties of North Carolina demonstrate higher achievement on average than students in the other parts of the state. These findings can be used to guide county level initiatives, such as school-based literacy programmes, to improve elementary education.
\end{abstract}

Keywords: Areal data; Bayesian analysis; Conditional auto-regressive prior; Education data; Finite mixture model; Multivariate spatial analysis

\section{Introduction}

In 2002, the US Congress enacted the No Child Left Behind Act requiring states to administer annual standardized tests to all students in federally funded schools (http: / / www2 ed. gov/policy/elsec/leg/esea02/index.html). In North Carolina, these tests are known as end-of-grade (EOG) tests. The EOG tests measure student performance on gradebased goals, objectives and competencies as set forth by the state's education department (North Carolina Department of Public Instruction, 2006). In particular, the mathematics tests measure competency in areas such as arithmetic operations, measurement and geometry, whereas the reading tests measure competency in areas such as vocabulary and reading comprehension. The raw EOG scores are subsequently categorized into four achievement levels:

(a) insufficient mastery;

(b) inconsistent mastery;

Address for correspondence: Brian Neelon, Department of Biostatistics and Bioinformatics, Box 2721 Duke University Medical Center, Durham, NC 27710-2721, USA.

E-mail: brian.neelon@duke.edu 
(c) consistent mastery;

(d) superior performance

(North Carolina Department of Public Instruction, 2007, 2008). Results of EOG tests have important implications for both individual schools and school districts, as they may affect state and federal funding levels.

Because scores can vary across geographic areas, there has been growing interest in examining regional differences in test scores, both at the national and at the state level. North Carolina, like many other states, is working to close the gap between low performing schools and those meeting No Child Left Behind Act standards. Despite this goal, relatively few studies have examined geographic disparities in EOG performance in an effort to identify high and low performing schools and school districts. In fact, we found only one related study examining gender differences in test performance across large national census divisions (Pope and Sydnor, 2010). Thus, there remains a need for a comprehensive study of varying test performance across a refined geographic scale. By pinpointing schools that fail to meet adequate yearly standards set forth by the No Child Left Behind Act, state and local education officials can develop targeted interventions to improve school performance in the areas of most need. Directed efforts such as these provide new opportunities to close the achievement gap in EOG test scores.

With these goals in mind, researchers from Duke University and the University of Michigan have conducted a study to understand better the factors influencing variation in EOG scores among elementary school children from across North Carolina. As a first step, the investigators obtained mathematics and reading test scores for fourth graders from all 100 countries in the state following completion of the 2008 school year, the most recent year for which such data were available. The data were then georeferenced by residential address and subsequently linked at the county level to data from the 2005-2009 American Community Survey (US Census Bureau, 2010). The aims of the study were to examine statewide variation in EOG test scores and to identify individual and county level predictors of EOG performance.

From an analytic perspective, the EOG data posed several unique challenges. First, mathematics and reading scores tend to be highly correlated measures; we therefore needed a flexible spatial model that could account for both within-subject and within-county associations. We also wanted a model that could yield accurate predictions of average student performance for each county while inducing spatial smoothing among sparsely populated counties where predictions may be less reliable. And, finally, we wanted a model that was robust to region-specific departures from normality in light of the skewness that is observed in the data. This paper describes a new multivariate spatial mixture model specifically designed to address these multiple aims.

Our proposed model capitalizes on recent developments in spatial modelling of multivariate, areal-referenced data, i.e. data in which the spatial units consist of discrete regions of space such as counties. Modelling of such data typically proceeds by introducing a set of region-specific random effects, which are then linked via a multivariate conditionally auto-regressive (CAR) prior distribution (Mardia, 1988). Previous applications of joint spatial models for areal data have focused on normal responses (Gelfand and Vounatsou, 2003), count responses for disease mapping (Carlin and Banerjee, 2002; Jin et al., 2005; Zhang et al., 2009; Congdon, 2010) and categorical responses (Gelfand and Vounatsou, 2003; Wall and Liu, 2009).

In many applications, the response variables are continuous but non-normally distributed, either because of multimodality, heavy skewness or both. In such cases, mixture models can provide a flexible framework for modelling the response distribution and can improve model fit. 
There is a well-established literature on mixture models for non-spatial data (McLachlan and Peel, 2000; Frühwirth-Schnatter, 2006). In the spatial setting, several researchers have proposed mixture models for point-referenced data-i.e. data indexed by a set of specific geographic coordinates. Gelfand et al. (2005) used a Dirichlet process mixture model to examine precipitation measurements at fixed locations in southern France. Kottas and Sansó (2007) extended the approach by allowing the point locations to be random. Ji et al. (2009) used a similar Poisson point process mixture model to identify cell abundance patterns from fluorescent intensity images of lymphatic tissue. For multivariate point-referenced data, Reich and Fuentes (2007) proposed a semiparametric mixture model specified through a stick breaking process. In the areal setting, Green and Richardson (2002) and Lawson and Clark (2002) proposed univariate mixture models for mapping disease relative risks. More recently, Wall and Liu (2009) developed a spatial latent class model for multivariate binary data and modelled the latent class indicators by using a multinomial probit model with spatially correlated error terms.

We extend this work by developing a multivariate spatial finite mixture model for continuous, areal-referenced data. We introduce spatial random effects for each mixture component, as well as for the mixing weights, to allow the shape of the multivariate response distribution to vary in flexible ways across geographic regions and covariate profiles. As such, our model provides a practical approach to multivariate spatial density estimation. By integrating across this mixture density, one can obtain region-specific inferences and model-based predictions of interest. We adopt a Bayesian inferential approach and for posterior computation develop an efficient Markov chain Monte Carlo (MCMC) algorithm that combines closed form Gibbs and Metropolis steps.

The remainder of the paper is organized as follows: Section 2 describes the EOG testing data; Section 3 outlines the model proposed and discusses prior specification, posterior computation and model selection; Section 4 presents results from two simulation studies highlighting important features of the model; Section 5 applies the method to the EOG data; and the final section provides a discussion and directions for future work.

\section{The end-of-grade data}

Table 1 provides a summary of the EOG data. For our analysis, we restricted the sample to nonHispanic white and non-Hispanic black students because of small sample sizes in other race and ethnicity groups and the effect of English as a second language on early school performance. Of the 78380 students, roughly half were male, about a third were non-Hispanic black and just over $43 \%$ received free or reduced price lunch at school through a federal subsidy programme. The mathematics scores ranged from 319 to 373 with a median of 352, and the reading scores ranged from 313 to 370 with a median of 346. Approximately three-quarters of the students achieved consistent mastery or higher on the mathematics examination, and nearly $63 \%$ achieved consistent mastery or better on reading.

Fig. 1 presents a bivariate histogram of the raw mathematics and reading scores (Fig. 1(a)) as well as a histogram of the standardized residuals based on an ordinary least squares regression that included as predictors gender, race, enrolment in a free- or reduced price lunch programme and county median household income (Fig. 1(b)). The distribution of the residuals is skewed towards lower values, particularly for reading, and the kurtoses in both directions are slightly negative. The univariate Kolmogorov-Smirnov tests on the residuals rejected the null hypothesis of normality ( $p<0.01$ for both outcomes), suggesting that the bivariate response distribution might be better modelled as a low dimensional finite mixture of normal distributions rather than as a single bivariate normal distribution. 
Table 1. Summary statistics for the EOG data $(N=78380)$

\begin{tabular}{|c|c|c|}
\hline Variable & $\begin{array}{c}\text { Median (interquartile } \\
\text { range) }\end{array}$ & $n(\%)$ \\
\hline Mathematics score & $352(345,358)$ & \\
\hline Reading score & $346(339,353)$ & \\
\hline County median household income (\$) & $44319(39676,51110)$ & \\
\hline County sample size & $443(203,874)$ & \\
\hline Male & & $39555(50.47)$ \\
\hline Non-Hispanic black race $\dagger$ & & $25219(32.18)$ \\
\hline $\begin{array}{l}\text { Enrolled in free- or reduced price lunch } \\
\text { programme }\end{array}$ & & $33959(43.33)$ \\
\hline \multicolumn{3}{|l|}{ Mathematics achievement level } \\
\hline Insufficient mastery (score $\leqslant 335$ ) & & $4470(5.70)$ \\
\hline Inconsistent mastery (score 336-344) & & $15040(19.19)$ \\
\hline Consistent mastery (score $345-357$ ) & & 37956 (48.43) \\
\hline Superior performance (score $\geqslant 358$ ) & & $20914(26.68)$ \\
\hline \multicolumn{3}{|l|}{ Reading achievement level } \\
\hline Insufficient mastery (score $\leqslant 334$ ) & & $11439(14.59)$ \\
\hline Inconsistent mastery ( score 335-342) & & $17618(22.48)$ \\
\hline Consistent mastery (score $343-353$ ) & & $30879(39.40)$ \\
\hline Superior performance (score $\geqslant 354$ ) & & $18444(23.53)$ \\
\hline
\end{tabular}

$\dagger$ Non-Hispanic black race and free-lunch enrolment were coded as binary (yes-no) variables.

There is also substantial variation in test scores across the state. Fig. 2 shows the Studentized ordinary least squares residuals averaged by county for both mathematics and reading. The spatial pattern is similar for both mathematics and reading, with negative residuals clustering in the interior north-east, along the eastern southern border and in the westernmost counties, whereas pockets of positive residuals appear in the centre of the state and along the southern border in the west. This pattern suggests positive spatial auto-correlation in the residuals, violating the ordinary least squares assumption of independently distributed errors. This points to the need for a model that explicitly accounts for spatial dependence, since ignoring such spatial structure could lead to biased inferences and inaccurate assessments of parameter uncertainty.

\section{Spatial mixture model}

\subsection{Model specification}

To develop the multivariate spatial mixture model, we focus on the bivariate case. It is conceptually straightforward to extend the approach to three or more outcomes.

A very general specification of the bivariate spatial mixture model can be expressed as

$$
\begin{gathered}
\mathbf{y}_{i j} \mid \phi_{i}, \boldsymbol{\psi}_{i} \sim \sum_{k=1}^{K} \pi_{i j k} N_{2}\left(\boldsymbol{\eta}_{i j k}, \boldsymbol{\Sigma}_{k}\right), \\
\boldsymbol{\eta}_{i j k}=\left(\begin{array}{c}
\eta_{1 i j k} \\
\eta_{2 i j k}
\end{array}\right)=\mathbf{X}_{i j} \boldsymbol{\beta}_{k}+\mathbf{V}_{i} \boldsymbol{\alpha}_{k}+\boldsymbol{\phi}_{i k}, \\
\pi_{i j k}=\frac{\exp \left(\mathbf{x}_{i j}^{\prime} \gamma_{k}+\mathbf{v}_{i}^{\prime} \boldsymbol{\delta}_{k}+\psi_{i k}\right)}{\sum_{h=1}^{K} \exp \left(\mathbf{x}_{i j}^{\prime} \gamma_{h}+\mathbf{v}_{i}^{\prime} \boldsymbol{\delta}_{h}+\psi_{i h}\right)}, \quad i=1, \ldots, n, \quad j=1, \ldots, n_{i}, \quad k=1, \ldots, K,
\end{gathered}
$$




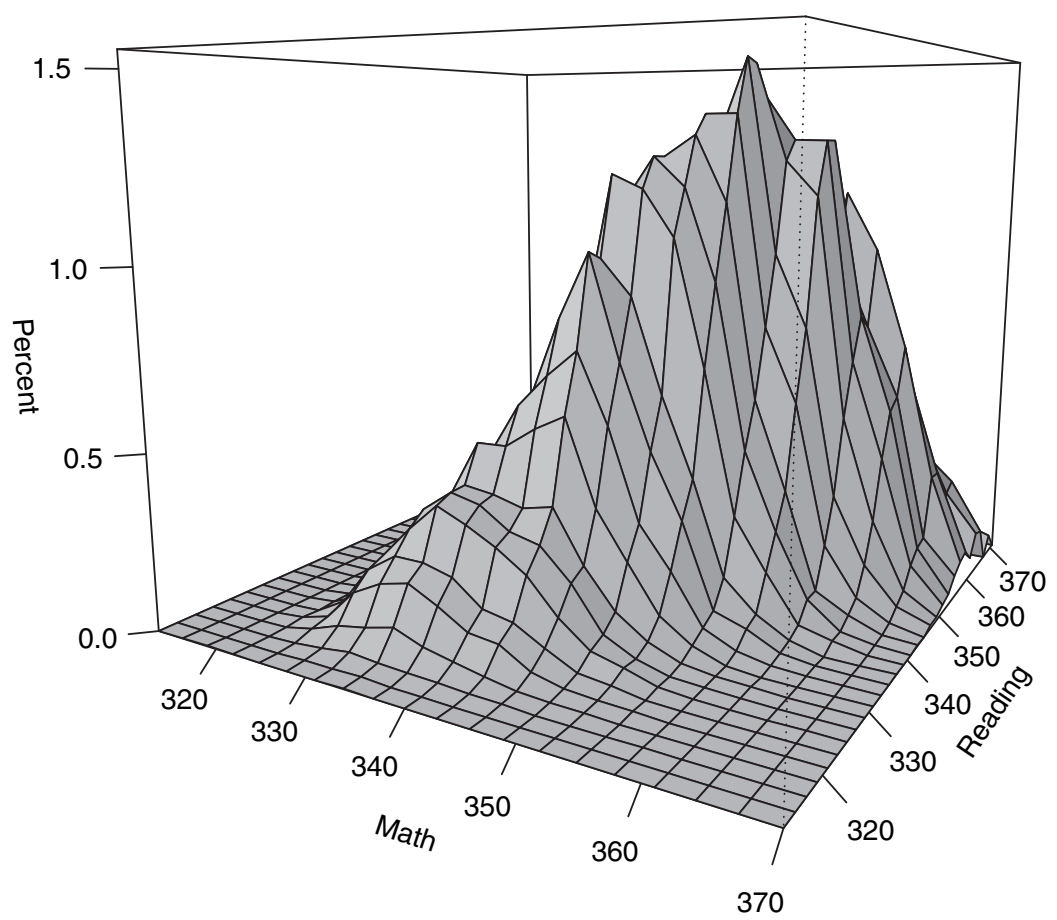

(a)

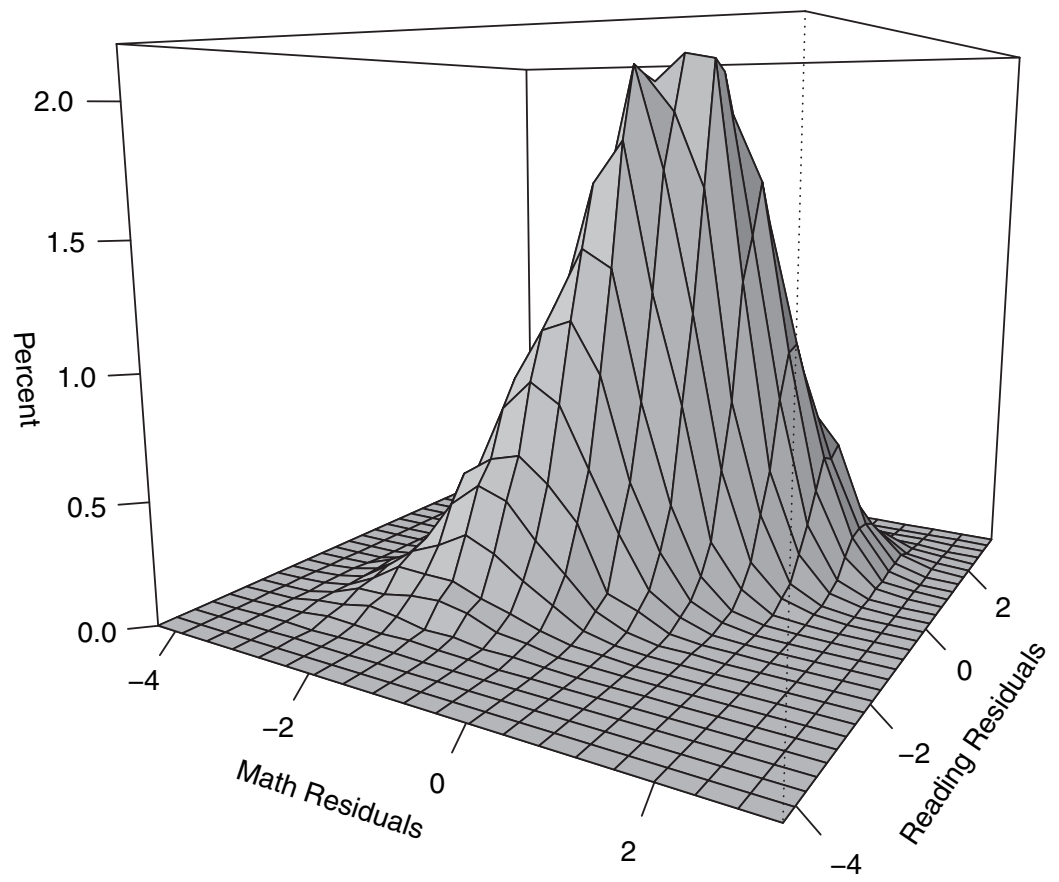

(b)

Fig. 1. Bivariate histogram of (a) the raw mathematics and reading scores and (b) ordinary least squares standardized residuals for the 2008 fourth-grade EOG test scores 


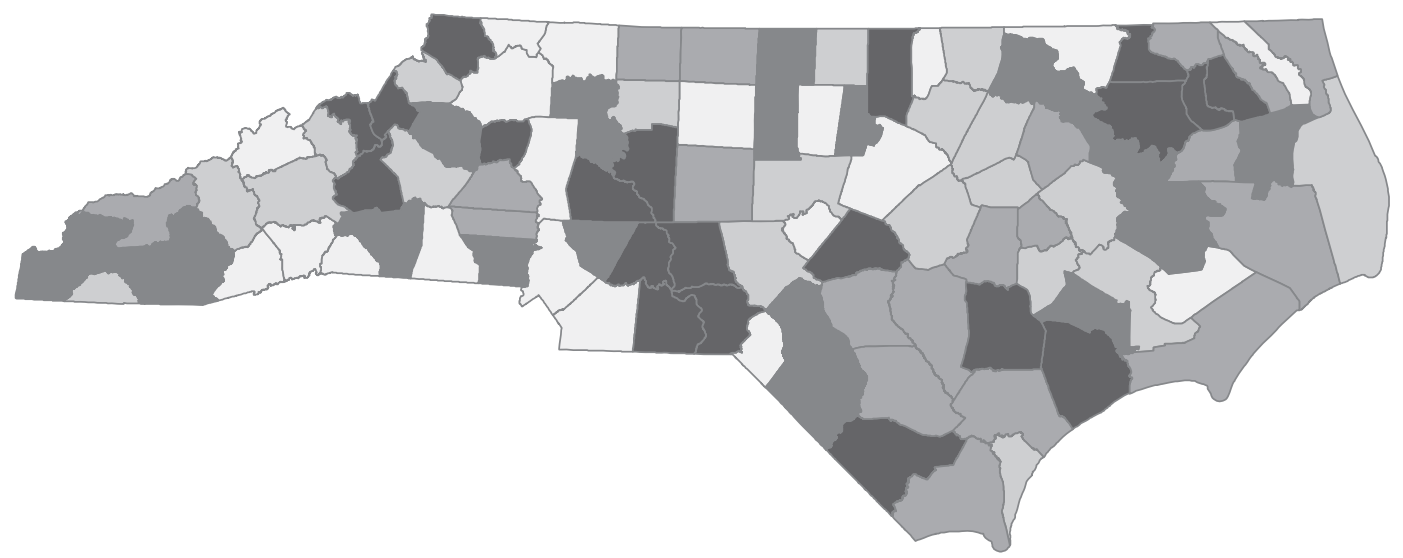

(a)

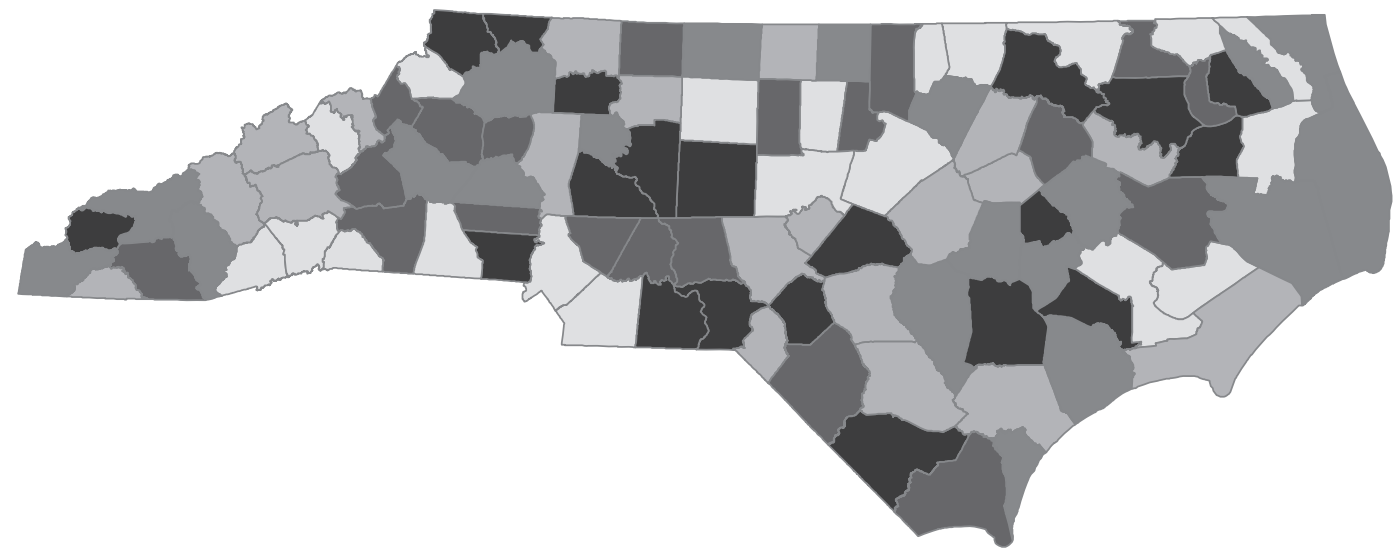

(b)

Fig. 2. Quintiles of county-averaged Studentized residuals for the 2008 fourth-grade EOG scores: (a) mathematics $(\square,-0.54--0.19 ; \square,-0.18--0.11 ; \square,-0.10--0.03 ; \square,-0.02-0.08 ; \square, 0.09-0.42$ ); (b) reading $(\square,-0.39--0.17 ; \square,-0.16--0.10 ; \square,-0.09-0.00 ; \square, 0.01-0.06 ; \square, 0.07-0.39)$

where $\mathbf{y}_{i j}=\left(y_{1 i j}, y_{2 i j}\right)^{\prime}$ denotes a $2 \times 1$ vector of mathematics and reading scores for the $j$ th student in the $i$ th county;

$$
\mathbf{X}_{i j}=\left(\begin{array}{cc}
\mathbf{x}_{i j}^{\prime} & \mathbf{0} \\
\mathbf{0} & \mathbf{x}_{i j}^{\prime}
\end{array}\right)
$$

is a $2 \times 2 p$ matrix of subject level covariates with corresponding $2 p \times 1$ component-specific fixed effects $\boldsymbol{\beta}_{k}=\left(\boldsymbol{\beta}_{1 k}^{\prime}, \boldsymbol{\beta}_{2 k}^{\prime}\right)^{\prime}$;

$$
\mathbf{V}_{i}=\left(\begin{array}{cc}
\mathbf{v}_{i}^{\prime} & \mathbf{0} \\
\mathbf{0} & \mathbf{v}_{i}^{\prime}
\end{array}\right)
$$

is a $2 \times 2 r$ matrix of county level covariates with corresponding $2 r \times 1$ component-specific fixed effects $\boldsymbol{\alpha}_{k}=\left(\boldsymbol{\alpha}_{1 k}^{\prime}, \boldsymbol{\alpha}_{2 k}^{\prime}\right)^{\prime} ; \phi_{i k}=\left(\phi_{1 i k}, \phi_{2 i k}\right)^{\prime}$ is a $2 \times 1$ vector of component-specific spatial random effects for the $i$ th county, with $\phi_{i}=\left(\phi_{i 1}^{\prime}, \ldots, \phi_{i K}^{\prime}\right)^{\prime} ; \Sigma_{k}$ is the component $k 2 \times 2$ variancecovariance matrix of $\mathbf{y}_{i j}$, conditional on $\phi_{i k} ; \gamma_{k}$ and $\delta_{k}$ are $p \times 1$ and $r \times 1$ vectors of mixing weight regression parameters with $\gamma_{1} \equiv \mathbf{0}$ and $\delta_{1} \equiv \mathbf{0}$ for identifiability; and $\psi_{i k}$ is a spatial random 
effect for county $i$ and mixing weight $k$, where $\psi_{i 1} \equiv 0$ and $\boldsymbol{\psi}_{i}=\left(\psi_{i 1}, \ldots, \psi_{i K}\right)^{\prime}$. Throughout, we assume the same set of covariates for the component means and the mixing weights, although in general this restriction is not necessary.

Model (1) is appealing because it allows the shape of the joint response distribution to change flexibly across spatial units and covariate levels. In particular, the within-component linear predictors (the $\boldsymbol{\eta}_{i j k} \mathrm{~s}$ ) permit the locations of the mixture components to vary throughout the population, whereas the mixing weight parameters allow the mass of the response distribution to shift in unique ways between individuals and counties. Together, these features produce distinct response distributions for each covariate profile and areal unit, thus allowing us to obtain county level predictions of EOG performance.

\subsection{Prior distributions}

For parameter estimation, we adopt a fully Bayesian approach, assuming prior distributions for all model parameters. First, to allow for spatial smoothing and borrowing of information across counties, for each $k$, we assign component-specific CAR priors (Besag, 1974; Besag et al., $1991)$ to the spatial random effects - a bivariate CAR prior for $\phi_{i k}$ and a univariate CAR prior for $\psi_{i k}$ :

$$
\begin{array}{rlrl}
\phi_{i k} \mid \phi_{(-i k)}, \boldsymbol{\Lambda}_{i k} \sim N_{2}\left(\xi_{k} \sum_{l \in \partial_{i}} \frac{w_{i l}}{w_{i+}} \phi_{l k}, \boldsymbol{\Lambda}_{i k}\right), & & k=1, \ldots, K, \\
\psi_{i k} \mid \psi_{(-i k)}, \tau_{i k}^{2} \sim N\left(\zeta_{k} \sum_{l \in \partial_{i}} \frac{w_{i l}}{w_{i+}} \psi_{l k}, \tau_{i k}^{2}\right), & k=2, \ldots, K,
\end{array}
$$

where $\partial_{i}$ denotes the set of neighbours for county $i, \xi_{k}$ and $\zeta_{k}$ are spatial smoothing parameters, $w_{i l}$ is an unnormalized proximity measure, $w_{i+}=\Sigma_{l \in \partial_{i}} w_{i l}, \boldsymbol{\Lambda}_{i k}=\boldsymbol{\Lambda}_{k} / w_{i+}$ is a component-specific scaled variance-covariance matrix for $\phi_{i k}$ conditional on $\phi_{(-i k)}$ and $\tau_{i k}^{2}=\tau_{k}^{2} / w_{i}$ is a componentspecific scaled variance parameter for $\psi_{i k}$. For the EOG study, we adopt intrinsic CAR priors to provide maximal smoothing of sparsely populated regions:

$$
\begin{gathered}
\phi_{i k} \mid \phi_{(-i k)}, \boldsymbol{\Lambda}_{k} \sim N_{2}\left(\frac{1}{m_{i}} \sum_{l \in \partial_{i}} \phi_{l k}, \frac{1}{m_{i}} \boldsymbol{\Lambda}_{k}\right), \\
\psi_{i k} \mid \psi_{(-i k)}, \tau_{k}^{2} \sim N\left(\frac{1}{m_{i}} \sum_{l \in \partial_{i}} \psi_{l k}, \frac{\tau_{k}^{2}}{m_{i}}\right),
\end{gathered}
$$

where $m_{i}$ denotes the number of neighbours sharing a geographic border with county $i$. Following Brook's lemma (see Banerjee et al. (2004)), priors (4) and (5) give rise to improper joint distributions for $\phi_{k}$ and $\psi_{k}$ :

$$
\begin{gathered}
\phi_{k} \mid \boldsymbol{\Lambda}_{k} \propto \exp \left[-\frac{1}{2} \phi_{k}^{\prime}\left\{(\mathbf{M}-\mathbf{A}) \otimes \boldsymbol{\Lambda}_{k}^{-1}\right\} \phi_{k}\right], \\
\boldsymbol{\psi}_{k} \mid \tau_{k}^{2} \propto \exp \left\{-\frac{1}{2 \tau_{k}^{2}} \boldsymbol{\psi}_{k}^{\prime}(\mathbf{M}-\mathbf{A}) \boldsymbol{\psi}_{k}\right\},
\end{gathered}
$$

where $\phi_{k}=\left(\phi_{1 k}^{\prime}, \ldots, \phi_{n k}^{\prime}\right)^{\prime}, \boldsymbol{\psi}_{k}=\left(\psi_{1 k}, \ldots, \psi_{n k}\right)^{\prime}, \mathbf{M}=\operatorname{diag}\left(m_{1}, \ldots, m_{n}\right)$ and $\mathbf{A}$ is an $n \times n$ adjacency matrix with $a_{i i}=0$ and $a_{i l}=1$ if counties $i$ and $l$ are neighbours, and $a_{i l}=0$ otherwise. Because (M-A) is singular, the joint distributions in expressions (6) and (7) are overparameterized and thus improper, although the conditional prior distributions given by equations (4) and (5) are themselves proper. Propriety of the posterior, when a fixed effect intercept is included in 
the model, is achieved by using a sum-to-0 constraint on the spatial random effects (Banerjee et al., 2004).

To ensure a well-identified model, we assign weakly informative proper priors to the remaining model parameters. For the within-component fixed effects, we assume exchangeable normal priors: $\boldsymbol{\beta}_{1 k}$ and $\boldsymbol{\beta}_{2 k} \sim N_{p}\left(\boldsymbol{\mu}_{\beta}, \boldsymbol{\Sigma}_{\beta}\right), \boldsymbol{\alpha}_{1 k}$ and $\boldsymbol{\alpha}_{2 k} \sim N_{r}\left(\boldsymbol{\mu}_{\alpha}, \boldsymbol{\Sigma}_{\alpha}\right)$ for $k=1, \ldots, K$. For the fixed effects within the mixing weights, $\gamma_{k}$ and $\boldsymbol{\delta}_{k}$, we assign $N_{p}\left(\boldsymbol{\mu}_{\gamma}, \boldsymbol{\Sigma}_{\gamma}\right)$ and $N_{r}\left(\boldsymbol{\mu}_{\delta}, \boldsymbol{\Sigma}_{\delta}\right)$ priors respectively, for $k=2, \ldots, K$. Throughout, we assume that the prior hyperparameters $\left(\boldsymbol{\mu}_{\beta}, \boldsymbol{\Sigma}_{\beta}\right.$, etc.) are identical across components, but in general this is not required. To complete the prior specification, we assign conjugate inverse Wishart IW $\left(\kappa_{0}, \mathbf{S}_{0}\right)$ and $\operatorname{IW}\left(\nu_{0}, \mathbf{D}_{0}\right)$ priors respectively to $\boldsymbol{\Sigma}_{k}$ and $\boldsymbol{\Lambda}_{k}$, and a conjugate inverse gamma $\operatorname{IG}(g, s)$ prior to $\tau_{k}^{2}(k=2, \ldots, K)$.

The model proposed accommodates a wide range of dependence structures. First, $\boldsymbol{\Sigma}_{12 k}$, the off-diagonal element of $\Sigma_{k}$, controls the component-specific within-subject association between outcomes. In the EOG study, for example, a positive value for $\boldsymbol{\Sigma}_{12 k}$ implies that, for component $k$, students with high mathematics scores also tend to have high reading scores conditional on the county level random effects. Similarly, $\boldsymbol{\Lambda}_{12 k}$, the off-diagonal element of $\boldsymbol{\Lambda}_{k}$, accounts for the component-specific, between-subject or within-region association between outcomes. In the EOG study, $\boldsymbol{\Lambda}_{12 k}>0$ implies that, for component $k$, counties with higher mean mathematics scores tend to have higher mean reading scores, adjusting for observed covariates. And, finally, the CAR priors on $\phi_{i k}$ and $\psi_{i k}$ capture associations between counties, implying that adjoining counties behave similarly with respect to their response distributions. Numerous submodels can be obtained by setting one or more of these association parameters to 0 . For example, setting $\Lambda_{12 k}=0 \forall k$ implies no between-subject or within-county association in responses. This is tantamount to assigning separate univariate CAR priors to $\phi_{1 i k}$ and $\phi_{2 i k}$. Further restricting $\Sigma_{12 k}$ to 0 for all $k$ would imply that there is no within-subject association between responses, and hence the outcomes are uncorrelated at all levels of the model.

\subsection{Posterior computation and model comparison}

Posterior inference proceeds via data augmentation by introducing a discrete latent labelling variable $C_{i j}$ that takes the value $k(k=1, \ldots, K)$ with probability $\pi_{i j k}$ defined in equation (1). Letting $\boldsymbol{\theta}_{k}=\left\{\boldsymbol{\beta}_{k}, \boldsymbol{\alpha}_{k}, \boldsymbol{\phi}_{k}, \boldsymbol{\Sigma}_{k}, \boldsymbol{\Lambda}_{k}\right\}$ denote the within-component parameters and $\boldsymbol{v}_{k}=\left\{\boldsymbol{\gamma}_{k}, \boldsymbol{\delta}_{k}, \boldsymbol{\psi}_{k}, \tau_{k}^{2}\right\}$ denote the mixing weight parameters, the joint posterior is given by

$$
\begin{aligned}
\pi\left(\boldsymbol{\theta}_{1}, \ldots, \boldsymbol{\theta}_{K}, \boldsymbol{v}_{2}, \ldots, \boldsymbol{v}_{K} \mid \mathbf{y}\right) \propto & \prod_{k=1}^{K}\left\{\prod_{i=1}^{n} \prod_{j=1}^{n_{i}}\left\{\pi_{i j k} N_{2}\left(\mathbf{y}_{i j} ; \boldsymbol{\eta}_{i j k}, \boldsymbol{\Sigma}_{k}\right)\right\}^{I_{\left(C_{i j}=k\right)}}\right. \\
& \left.\times \exp \left[-\frac{1}{2} \boldsymbol{\phi}_{k}^{\prime}\left\{(\mathbf{M}-\mathbf{A}) \otimes \boldsymbol{\Lambda}_{k}^{-1}\right\} \boldsymbol{\phi}_{k}\right] \pi\left(\boldsymbol{\beta}_{k}\right) \pi\left(\boldsymbol{\alpha}_{k}\right) \pi\left(\boldsymbol{\Sigma}_{k}\right) \pi\left(\boldsymbol{\Lambda}_{k}\right)\right\} \\
& \times \prod_{h=2}^{K} \exp \left\{-\frac{1}{2 \tau_{h}^{2}} \boldsymbol{\psi}_{h}^{\prime}(\mathbf{M}-\mathbf{A}) \boldsymbol{\psi}_{h}\right\} \pi\left(\boldsymbol{\gamma}_{h}\right) \pi\left(\boldsymbol{\delta}_{h}\right) \pi\left(\tau_{h}^{2}\right)
\end{aligned}
$$

where $I(\cdot)$ denotes the indicator function and the $\pi(\cdot)$ s represent the prior distributions for their respective parameters, as described in the previous section.

For posterior computation, we propose an MCMC algorithm that combines draws from full conditionals with Metropolis-based updates. After assigning initial values to the model parameters, the algorithm iterates between the following steps:

(a) for $k=2, \ldots, K$, update $\gamma_{k}$ and $\delta_{k}$ by using random-walk Metropolis steps;

(b) for $k=2, \ldots, K$ and $i=1, \ldots, n$, update $\psi_{i k}$ by using random-walk Metropolis steps;

(c) for $k=2, \ldots, K$, update $\tau_{k}^{2}$ from its closed form full conditional distribution; 
(d) for all $(i, j)$, sample the mixture component indicators from a discrete distribution taking values $\{k=1, \ldots, K\}$ with posterior probabilities $\left\{p_{i j 1}, \ldots, p_{i j K}\right\}$ as described in Appendix A;

(e) for $k=1, \ldots, K$, sample the component-specific parameters $\boldsymbol{\beta}_{1 k}, \boldsymbol{\beta}_{2 k}, \boldsymbol{\alpha}_{1 k}, \boldsymbol{\alpha}_{2 k}, \boldsymbol{\Sigma}_{k}, \boldsymbol{\Lambda}_{k}$ and $\phi_{i k}(i=1, \ldots, n)$ from their closed form full conditionals;

(f) at the end of each MCMC iteration, apply a sum-to-0 constraint to $\phi_{k}(k=1, \ldots, K)$ and $\psi_{k}(k=2, \ldots, K)$.

Explicit details of the algorithm are provided in Appendix A. An appealing feature of the MCMC algorithm is that the within-component regression parameters and spatial effects have convenient closed form full conditionals, leading to straightforward and efficient posterior sampling. Only the mixing weight parameters require Metropolis-based updates.

Convergence is monitored by running multiple chains from dispersed initial values and performing standard Bayesian diagnostics, such as trace plots and evaluation of the BrooksGelman-Rubin statistic (Gelman et al., 2004). Careful attention to such diagnostics is especially important for complex latent variable models to ensure parameter identifiability. In our experience, the MCMC algorithm proposed is generally robust to choices of initial values, with the possible exception of the mixing parameters $\gamma$ and $\delta$, which can be slow to converge for poorly chosen starting values. One way to choose initial values for these parameters is to perform a $K$-level cluster analysis, to fit a multinomial logit regression to the resulting cluster indicators and to use the ensuing parameter estimates as starting values.

A well-known computational challenge for Bayesian finite mixture models is 'label switching' in which draws of component-specific parameters may be associated with different component labels during the course of the MCMC run. Consequently, component-specific posterior summaries that average across the draws will be invalid. As a solution, Stephens (2000) proposed a post hoc relabelling algorithm based on a Kullback-Leibler divergence function. We apply this approach for the analysis of the EOG data that are described in Section 5.

For model comparison, we adopt the deviance information criterion DIC that was proposed by Spiegelhalter et al. (2002). DIC includes a goodness-of-fit term along with a penalty for model complexity. Models with smaller DIC are considered preferable. For the EOG application, we apply a modified version of DIC that was recommended by Celeux et al. (2006) for finite mixture models. This modified DIC, termed $\mathrm{DIC}_{3}$, uses the posterior predictive density of $\mathbf{y}$ to estimate the penalty term and is closely related to a measure put forward by Richardson (2002) to avoid overfitting the number of mixture components.

Several approaches can be used to determine the number of classes, $K$. One pragmatic approach (and the one adopted here) is to impose an upper bound (say, $K_{\max }$ ) on the number of classes, and then to use a model selection criterion such as DIC to choose the optimal value of $K=1, \ldots, K_{\max }$. In our application, we set $K_{\max }=2$ because we hypothesized the existence of at most two latent classes within the student population (namely 'high' and 'low' performers), and because inspection of the unimodal residual plot in Fig. 1(b) suggested that a low dimensional mixture would adequately capture important features of the data. A more formal - albeit computationally challenging _ approach would be to treat $K$ as a random variable and to employ either a dimension switching MCMC algorithm (Green, 1995) or to recast the model as an infinite mixture vis-à-vis a stick breaking representation (Sethuraman, 1994). We consider extensions to infinite mixtures further in Section 6.

\section{Simulation studies}

To examine the performance of our model, we conducted two simulation studies. For the first 
study, we generated 200 data sets from a basic two-component mixture model without covariates. Our aims here were threefold:

(a) to evaluate MCMC performance,

(b) to ensure that we obtained reasonable parameter estimates under the true model and

(c) to highlight the model as a practical approach to spatial density estimation whereby the shape of the response distribution is allowed to vary flexibly across spatial units.

To emulate the EOG data, we used the North Carolina county level adjacency matrix for the simulation. This matrix contains 512 adjacencies between the 100 North Carolina counties. For the purposes of the simulation, counties were labelled from '1' to '100'. Because the (M-A) matrix in equations (6) and (7) is singular, the spatial random effects cannot be simulated directly. We therefore introduced the spatial smoothing parameters $\xi$ and $\zeta$, defined in equations (2) and (3), and set them equal to $1-10^{-6}$. We then generated spatial random effects according to joint distributions (6) and (7) augmented with the smoothing parameters. Next, for each county, we simulated 80 mathematics and reading scores from the two-component mixture model

$$
\begin{gathered}
\mathbf{y}_{i j} \mid \boldsymbol{\phi}_{i}, \boldsymbol{\psi}_{i} \sim \sum_{k=1}^{2} \pi_{i j k} N_{2}\left\{\left(\begin{array}{c}
\beta_{10 k}+\phi_{1 i k} \\
\beta_{20 k}+\phi_{2 i k}
\end{array}\right), \boldsymbol{\Sigma}_{k}\right\}, \\
\operatorname{logit}\left(\pi_{i j 2}\right)=\gamma_{0}+\psi_{i}, \quad i=1, \ldots, 100, \quad j=1, \ldots, 80,
\end{gathered}
$$

where $\pi_{i j 2}$ denotes the weight for the second mixture component. For $k=1,2$ we assigned independent $N(0,1000)$ priors to $\beta_{10 k}$ and $\beta_{20 k}$, and $\operatorname{IW}\left(3, \mathbf{I}_{2}\right)$ priors to $\boldsymbol{\Sigma}_{k}$ and $\boldsymbol{\Lambda}_{k}$; for $\gamma_{0}$, we assigned an $N(0,1000)$ prior; for $\phi_{i k}=\left(\phi_{1 i k}, \phi_{2 i k}\right)^{\prime}$ and $\psi_{i}$, we assigned bivariate and univariate

Table 2. Average posterior estimates and $95 \%$ coverage probabilities across 200 simulated

\begin{tabular}{|c|c|c|c|c|c|}
\hline \multirow{2}{*}{$\begin{array}{l}\text { Mixture } \\
\text { component }\end{array}$} & \multirow[t]{2}{*}{ Parameter } & \multirow[t]{2}{*}{ Description } & \multicolumn{3}{|c|}{ Average posterior } \\
\hline & & & $\begin{array}{l}\text { True } \\
\text { value }\end{array}$ & Mean & $\begin{array}{l}95 \% \\
\text { coverage }\end{array}$ \\
\hline \multirow[t]{8}{*}{1} & $\beta_{101}$ & Mathematics intercept & 340 & 340.01 & 0.93 \\
\hline & $\beta_{201}$ & Reading intercept & 330 & 330.02 & 0.95 \\
\hline & $\Sigma_{111}$ & $\operatorname{var}\left(y_{1 i j} \mid \phi_{1 i 1}\right)$ & 20 & 20.10 & 0.96 \\
\hline & $\Sigma_{121}$ & $\operatorname{cov}\left(y_{1 i j}, y_{2 i j} \mid \phi_{i 1}\right)$ & 10 & 10.17 & 0.94 \\
\hline & $\Sigma_{221}$ & $\operatorname{var}\left(y_{2 i j} \mid \phi_{2 i 1}\right)$ & 36 & 36.34 & 0.95 \\
\hline & $\Lambda_{111}$ & $\operatorname{var}\left(\phi_{1 i 1}\right)$ & 9 & 8.77 & 0.96 \\
\hline & $\Lambda_{121}$ & $\operatorname{cov}\left(\phi_{1 i 1}, \phi_{2 i 1}\right)$ & 3 & 2.80 & 0.95 \\
\hline & $\Lambda_{221}$ & $\operatorname{var}\left(\phi_{2 i 1}\right)$ & 4 & 3.49 & 0.91 \\
\hline \multirow[t]{8}{*}{2} & $\beta_{102}$ & Mathematics intercept & 360 & 360.01 & 0.97 \\
\hline & $\beta_{202}$ & Reading intercept & 345 & 345.01 & 0.96 \\
\hline & $\Sigma_{112}$ & $\operatorname{var}\left(y_{1 i j} \mid \phi_{1 i 2}\right)$ & 50 & 50.04 & 0.96 \\
\hline & $\Sigma_{122}$ & $\operatorname{cov}\left(y_{1 i j}, y_{2 i j} \mid \phi_{i 2}\right)$ & 20 & 19.99 & 0.93 \\
\hline & $\Sigma_{222}$ & $\operatorname{var}\left(y_{2 i j} \mid \phi_{2 i 2}\right)$ & 40 & 40.10 & 0.96 \\
\hline & $\Lambda_{112}$ & $\operatorname{var}\left(\phi_{1 i 2}\right)$ & 4 & 3.53 & 0.93 \\
\hline & $\Lambda_{122}$ & $\operatorname{cov}\left(\phi_{1 i 2}, \phi_{2 i 2}\right)$ & 6 & 5.73 & 0.92 \\
\hline & $\Lambda_{222}$ & $\operatorname{var}\left(\phi_{2 i 2}\right)$ & 16 & 15.74 & 0.93 \\
\hline \multirow{2}{*}{$\begin{array}{l}\text { Mixing weight } \\
\text { parameters }\end{array}$} & $\gamma_{0}$ & Mixing weight intercept & 0.75 & 0.75 & 0.96 \\
\hline & $\tau^{2}$ & $\operatorname{var}\left(\psi_{i}\right)$ & 1 & 1.00 & 0.94 \\
\hline
\end{tabular}
data sets 


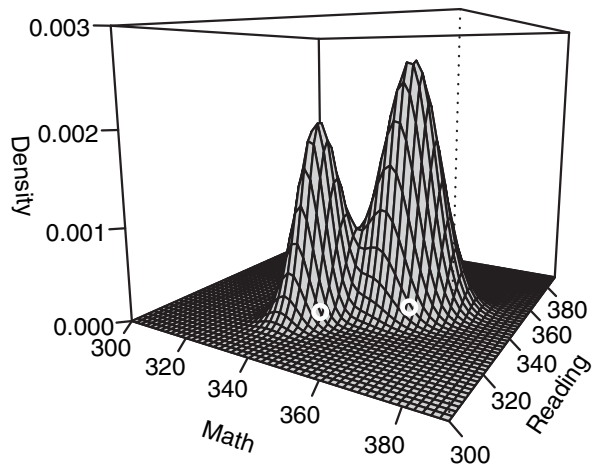

(a)

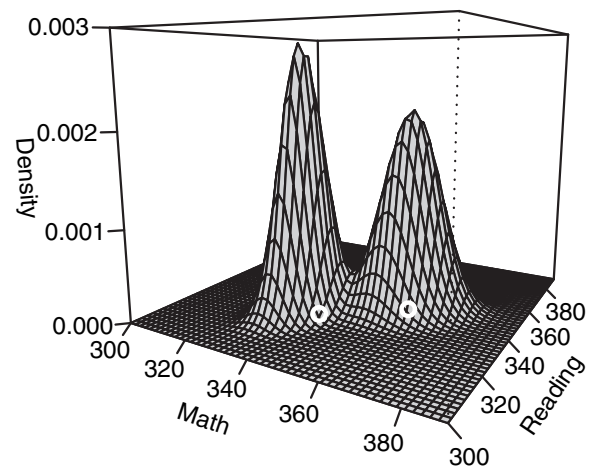

(c)

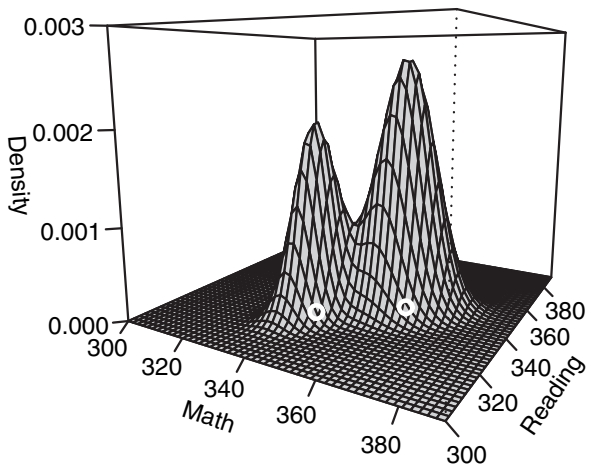

(b)

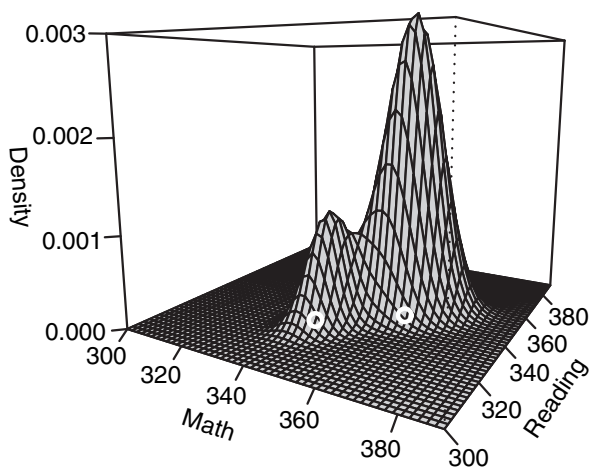

(d)

Fig. 3. True and estimated posterior densities from a randomly selected data set from the first simulation study (O, true component means: $\boldsymbol{\beta}_{01}=(340,330)^{\prime}$ and $\left.\boldsymbol{\beta}_{02}=(360,345)^{\prime}\right)$ : (a) true simulated density for an 'average' county (random effects set equal to 0); (b) corresponding model-estimated density; (c) estimated density for a county (county 31 ) in which the components split apart; (d) estimated density for a county (county 100) in which the component locations shift towards higher values and more mass is concentrated on the upper component

intrinsic CAR priors respectively; and, for $\tau^{2}$, the conditional variance of $\psi_{i}$, we assigned an $\mathrm{IG}(0.01,0.01)$ prior.

For each simulation, we ran 2000 MCMC iterations in R version 2.14 (R Development Core Team, 2011) using a burn-in of 1000, which was sufficient to ensure convergence based on standard diagnostics. To avoid label switching, we simulated extremely well-separated mixture components, effectively imposing the order constraints $\beta_{101}<\beta_{102}$ and $\beta_{201}<\beta_{202}$.

Table 2 presents the posterior means, averaged across the 200 simulations, along with $95 \%$ coverage rates and the true parameter values used to generate the data. The posterior estimates showed minimal bias, and the coverage rates were near the nominal values for all parameters. Fig. 3 displays the bivariate densities from a randomly selected simulation study. Fig. 3(a) shows the true (i.e. simulated) density for an 'average' county in which the random effects were set to 0. Fig. 3(b) displays the corresponding model-estimated density, and Figs 3(c) and 3(d) show the estimated densities for two randomly selected counties. The white circles denote the true component means: $\boldsymbol{\beta}_{01}=(340,330)^{\prime}$ and $\boldsymbol{\beta}_{02}=(360,345)^{\prime}$. As expected, the estimated average density in Fig. 3(b) closely mirrors the true density. Fig. 3(c) shows a county in which the mixture components diverge and there is a shift in mass towards the lower component. In Fig. 


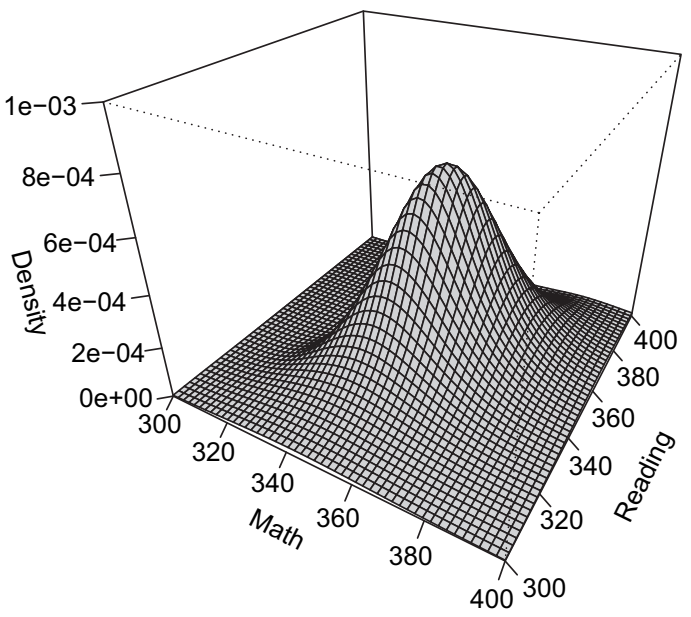

(a)

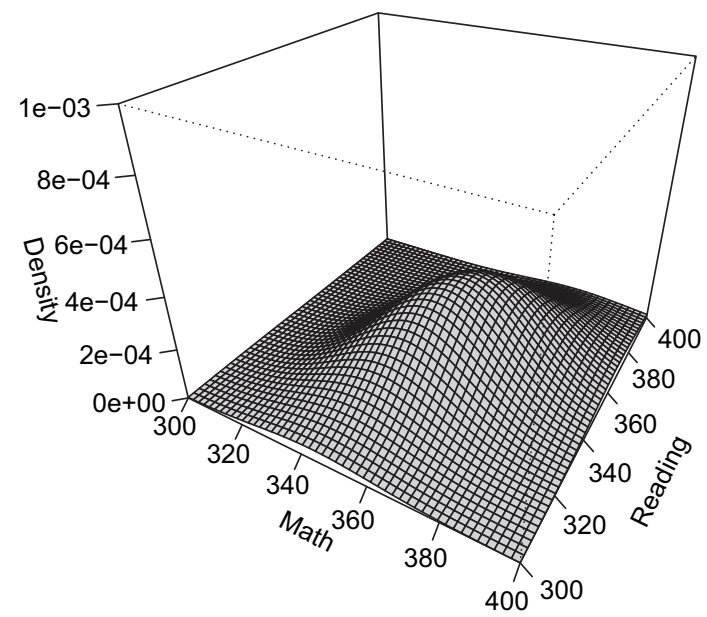

(c)

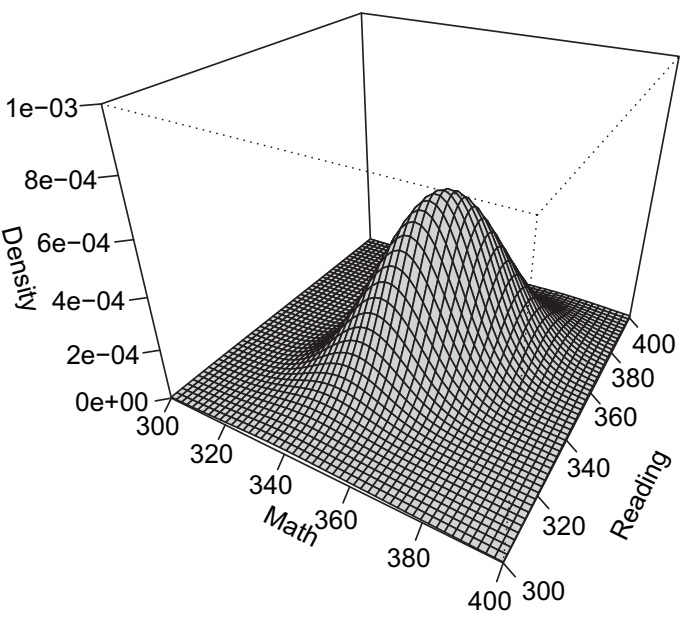

(b)

Fig. 4. True and estimated bivariate densities for simulation study 2: (a) true 5 degrees-of-freedom bivariate skew $t$-distribution; (b) estimated density for an 'average' county (random effects set to 0 ) from the two-component mixture model; (c) estimated density for an average county from the one-component model

3(d), the component locations shift towards higher mathematics and reading values and the mass is concentrated on the upper component.

Whereas we expect the proposed mixture model to perform well for multimodal data, one might wonder whether the same holds for unimodal but skewed data such as the EOG scores. To address this question, we conducted a second simulation in which we generated data from a 5 degrees-of-freedom bivariate skew $t$-distribution with location $\boldsymbol{\beta}_{0}=(350,360)^{\prime}$, skewness $\boldsymbol{\lambda}=(2,-2)^{\prime}$ and scale

$$
\boldsymbol{\Sigma}=\left(\begin{array}{cc}
300 & 50 \\
50 & 400
\end{array}\right)
$$

This parameterization yielded a bivariate distribution that is similar to the empirical histogram of EOG scores shown in Fig. 1(b). As in the first simulation study, we used the North Carolina 


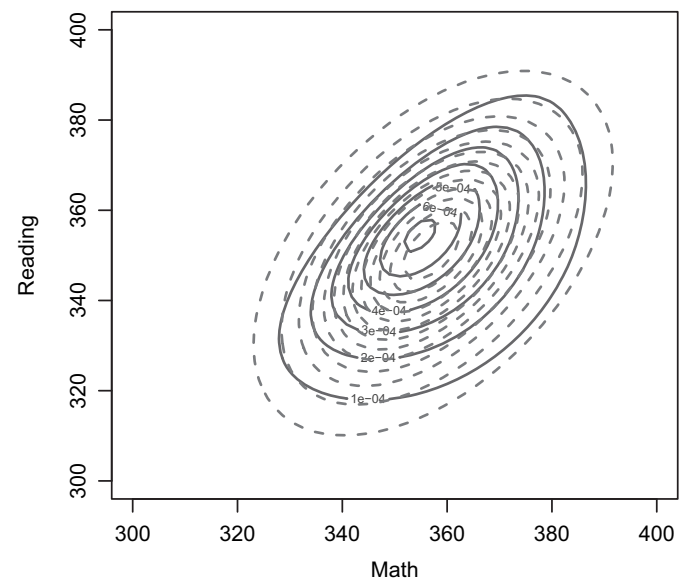

(a)

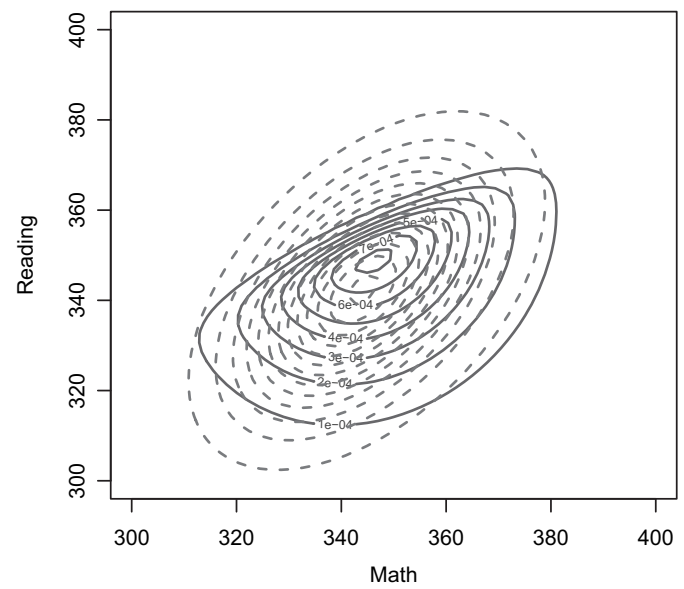

(c)

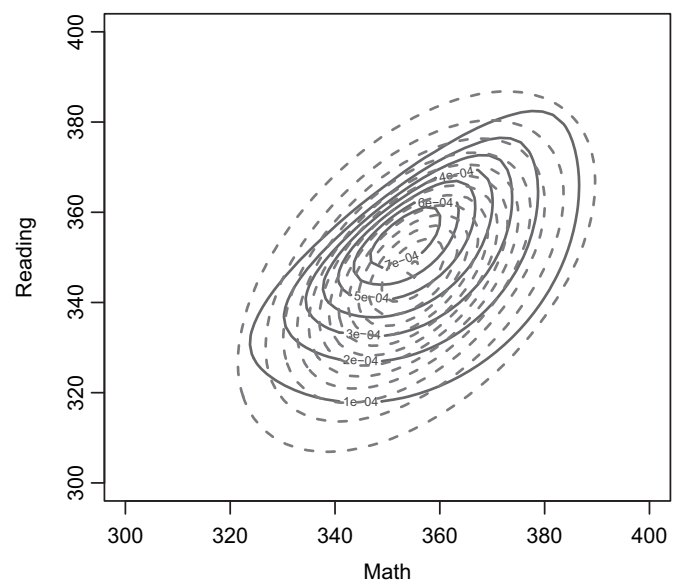

(b)

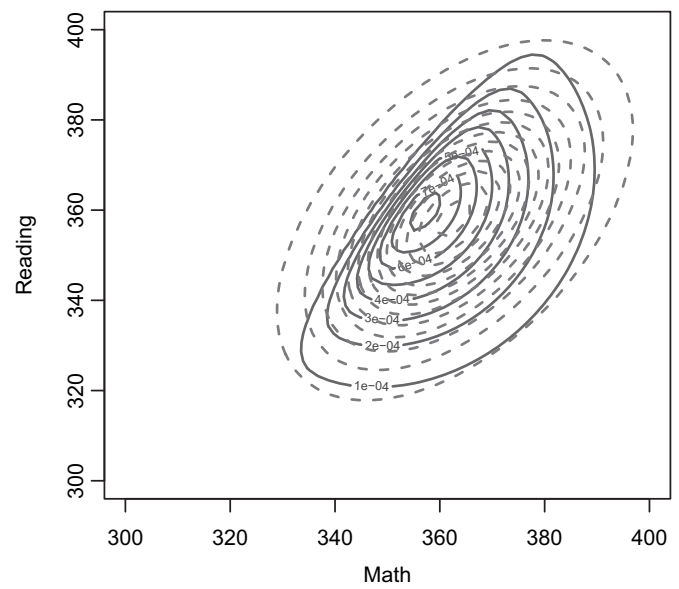

(d)

Fig. 5. Contour plots for (a) an 'average' county and (b)-(d) selected simulated counties ( - , true densities; - - - , estimated contours from the two-component model): (b) county 18; (c) county 85; (d) county 94

adjacency matrix and generated 80 observations from each of the 100 counties. Using bivariate CAR random effects, we allowed the location and skewness parameters to vary across the counties. We then fitted the two-component model given in equation (9). For comparison, we also fitted a single-component bivariate normal spatial model, which might be regarded as a conventional approach to analysing such data. We also computed DIC-statistics to compare the one- and two-component models further.

Fig. 4 presents the true bivariate density (Fig. 4(a)) along with the estimated 'average' densities (random effects set to 0) from the two-component (Fig. 4(b)) and one-component (Fig. 4(c)) models. Compared with the one-component model, the two-component model could reproduce the true density reasonably well, although even the two-component model failed to capture fully the kurtosis that is displayed in Fig. 4(a). The one-component model, in contrast, was unduly influenced by the tails of the skew $t$-distribution; as a result, its mass is far more dispersed than that of the two-component model. In terms of DIC, the two-component model vastly 
outperformed the one-component model (139487 versus 142566 respectively), confirming that the method proposed provides a superior fit over the conventional approach even for unimodal skewed data.

Fig. 5 presents contour plots comparing the true and two-component estimated densities, both overall and for selected counties. As Fig. 5(a) indicates, the two-component model accurately estimated the location of the true density, although with greater dispersion. In Figs 5(b)-5(d), the two-component model identified the location shifts in the county-specific densities but could not model the extreme skewness that is exhibited in Figs 5(c) and 5(d). Although a higher order mixture might provide a better fit, identifiability problems could arise in attempting to fit a high dimensional mixture to unimodal data. It is also possible that, with only 80 replicates per county in the simulation study, there was insufficient sample size to capture all aspects of the countyspecific densities. Ultimately, however, mixtures based on multivariate normal disturbutions may lack the flexibility that is required to model extremely skewed data. An alternative might be to develop a model based on mixtures of skew elliptical distributions, such as skew normal or skew $t$-densities. We consider this extension further in Section 6.

\section{Analysis of the end-of-grade data}

Next, we fit the following two-component spatial mixture model to the EOG data:

$$
\begin{gathered}
\mathbf{y}_{i j} \mid \phi_{i}, \boldsymbol{\psi}_{i} \sim \sum_{k=1}^{2} \pi_{i j k} N_{2}\left\{\left(\begin{array}{c}
\eta_{1 i j k} \\
\eta_{2 i j k}
\end{array}\right), \boldsymbol{\Sigma}_{k}\right\}, \\
\eta_{1 i j k}=\beta_{10 k}+\beta_{11 k} \times \mathrm{Male}_{i j}+\beta_{12 k} \times \mathrm{NHB}_{i j}+\beta_{13 k} \times \text { Freelunch }_{i j}+\alpha_{11 k} \times \mathrm{Medinc}_{i}+\phi_{1 i k}, \\
\eta_{2 i j k}=\beta_{20 k}+\beta_{21 k} \times \mathrm{Male}_{i j}+\beta_{22 k} \times \mathrm{NHB}_{i j}+\beta_{23 k} \times \text { Freelunch }_{i j}+\alpha_{21 k} \times \text { Medinc }_{i}+\phi_{2 i k}, \\
\operatorname{logit}\left(\pi_{i j 2}\right)=\gamma_{0}+\gamma_{1} \times \mathrm{Male}_{i j}+\gamma_{2} \times \mathrm{NHB}_{i j}+\gamma_{3} \times \text { Freelunch }_{i j}+\delta_{1} \times \text { Medinc }_{i}+\psi_{i}, \\
i=1, \ldots, 100, \quad j=1, \ldots, n_{i}, \quad k=1,2,
\end{gathered}
$$

where $\mathbf{y}_{i j}$ is a vector of mathematics and reading scores for the $j$ th subject in the $i$ th county, Male is a dichotomous indicator of male gender, NHB is a dichotomous indicator taking the value 1 if the student is non-Hispanic black and 0 if non-Hispanic white, Freelunch is a dichotomous indicator taking the value 1 if the student participated in a free- or reduced price lunch programme and 0 otherwise, Medinc denotes county median household income (in thousands of dollars) and $\pi_{i j 2}$ denotes the weight for the second mixture component.

As in the simulation studies, we assigned a bivariate CAR prior to $\phi_{i k}$ and a univariate CAR prior to $\psi_{i}$. We assumed weakly informative proper priors for all other model parameters: for $\boldsymbol{\beta}_{1 k}=\left(\beta_{10 k}, \beta_{11 k}, \beta_{12 k}, \beta_{13 k}\right)^{\prime}, \boldsymbol{\beta}_{2 k}=\left(\beta_{20 k}, \beta_{21 k}, \beta_{22 k}, \beta_{23 k}\right)^{\prime}$ and $\gamma=\left(\gamma_{0}, \gamma_{1}, \gamma_{2}, \gamma_{3}\right)^{\prime}$, we assigned conjugate $N_{4}\left(0,1000 \mathbf{I}_{4}\right)$ priors; for $\alpha_{11 k}, \alpha_{21 k}$ and $\delta_{1}$, we assigned $N(0,1000)$ priors; for $\Sigma_{k}$ and $\boldsymbol{\Lambda}_{k}$, we assigned $\operatorname{IW}\left(3, \mathbf{I}_{2}\right)$ priors; and for $\tau^{2}$ we assigned an $\operatorname{IG}(0.01,0.01)$ prior. We ran two initially dispersed chains for 20000 iterations each, discarding the first 10000 as burn-in. To reduce auto-correlation, we retained every 10 th iteration.

Model diagnostics indicated efficient mixing and rapid convergence of the chains. Fig. 6 presents the post-burn-in trace plots for four selected model parameters: $\beta_{111}$, the component 1 mathematics coefficient for male gender; $\gamma_{3}$, the mixing weight coefficient for Freelunch; $\tau^{2}$, the variance of $\psi_{i}$; and $\boldsymbol{\Lambda}_{222}$, the variance of $\phi_{2 i 2}$. The chains overlapped substantially, with no evidence of label switching within individual chains, and hence Stephens's (2000) relabelling algorithm converged rapidly. The component labels did require reordering across chains, but the proper labelling was easily identified so that the chains could be combined for summary 


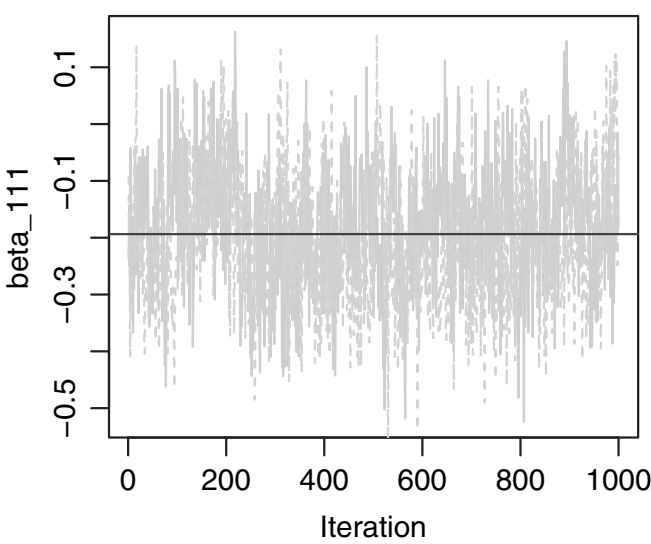

(a)

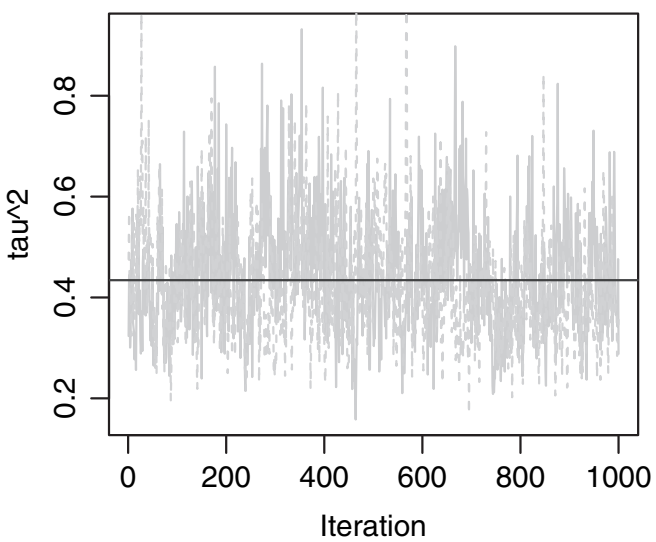

(c)

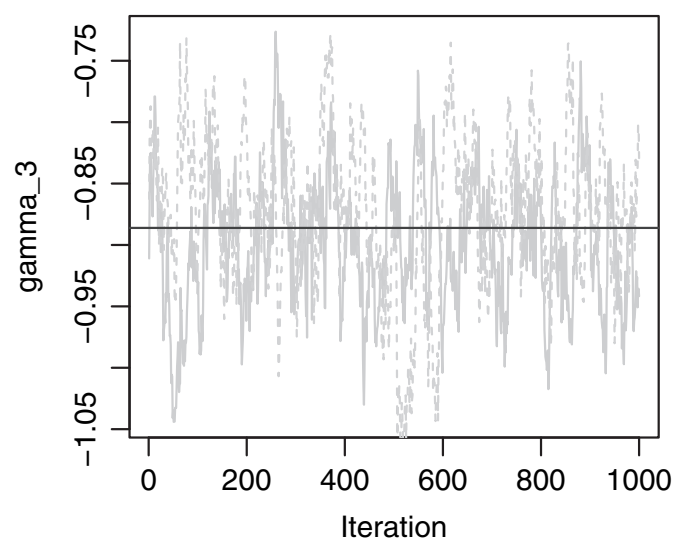

(b)

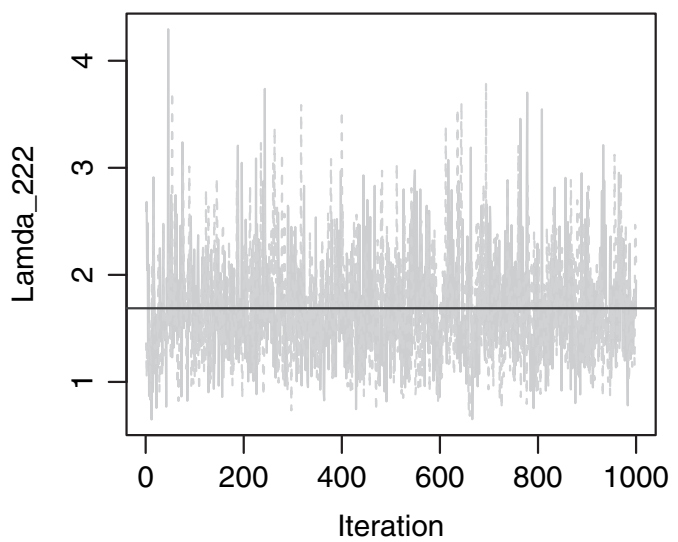

(d)

Fig. 6. Post-burn-in Markov chain Monte Carlo trace plots for four parameters from the proposed model (|, chain 1 ; , chain 2 ; - combined posterior mean): (a) $\beta_{111}$, the component 1 mathematics coefficient for male gender (Brooks-Gelman-Rubin upper credible interval 1.09); (b) $\gamma_{3}$, the mixing weight coefficient for Freelunch (Brooks-Gelman-Rubin upper credible interval 1.21); (c) $\tau^{2}$, the variance of $\psi_{i}$ (Brooks-Gelman-Rubin upper credible interval 1.13); (d) $\Lambda_{222}$, the variance of $\phi_{2 i 2}$ (Brooks-Gelman-Rubin upper credible interval 1.00)

purposes. For each of the four parameters, the Brooks-Gelman-Rubin upper credible interval was 1.21 or less, indicating adequate convergence of the chains.

To evaluate the performance of our model, we compared our proposed model with three submodels:

(a) a one-component version of the proposed spatial mixture model with a bivariate CAR prior for $\phi_{i}$ (submodel 1),

(b) a two-component fixed effects model excluding $\phi_{i k}$ and $\psi_{i}$ (submodel 2) and

(c) a two-component model with component-specific $\phi_{i k}$ but no $\psi_{i}$ (submodel 3).

Table 3 provides the model comparison results for the various models. The two-component random-effects models substantially outperformed submodels 1 and 2 . Overall, the full model had the best performance, suggesting that incorporating the random effects $\psi_{i}$ in the mixing weights provided a modest additional benefit relative to submodel 3 . 
Table 3. Model comparison statistics for analysis of the EOG data

\begin{tabular}{|lrrrr|}
\hline Model description & $\bar{D}$ & $p_{D}$ & \multicolumn{1}{c}{$D I C_{3}$} & $\Delta$ \\
\hline One-component model & & & & \\
Two-component fixed effects model & 1068553 & 174 & 1068727 & - \\
Two-component model excluding $\psi_{i}$ & 1065144 & 28 & 1067283 & 1444 \\
Proposed model & 1064990 & 328 & 1065439 & 1844 \\
& & & & 121 \\
\hline
\end{tabular}

Table 4. Posterior means and $95 \%$ credible intervals for the proposed model

\begin{tabular}{|c|c|c|c|c|}
\hline $\begin{array}{l}\text { Mixture } \\
\text { component }\end{array}$ & Parameter & Description & $\begin{array}{l}\text { Posterior } \\
\text { mean }\end{array}$ & $\begin{array}{l}95 \% \text { credible } \\
\text { interval }\end{array}$ \\
\hline \multirow[t]{17}{*}{$1(58 \%)$} & $\beta_{101}$ & Mathematics intercept & 349.25 & {$[348.67,349.77]$} \\
\hline & $\beta_{111}$ & Male & -0.19 & {$[-0.41,0.05]$} \\
\hline & $\beta_{121}$ & NHB race & -3.12 & {$[-3.45,-2.77]$} \\
\hline & $\beta_{131}$ & Free or reduced lunch & -2.50 & {$[-2.83,-2.18]$} \\
\hline & $\alpha_{111}$ & Median household income $(\$ 1000)$ & 0.03 & {$[-0.01,0.08]$} \\
\hline & $\beta_{201}$ & Reading intercept & 344.50 & {$[343.84,345.07]$} \\
\hline & $\beta_{211}$ & Male & -1.74 & {$[-1.98,-1.49]$} \\
\hline & $\beta_{221}$ & NHB race & -2.95 & {$[-3.31,-2.56]$} \\
\hline & $\beta_{231}$ & Free or reduced lunch & -2.98 & {$[-3.32,-2.62]$} \\
\hline & $\alpha_{211}$ & Median household income ( $\$ 1000)$ & 0.05 & {$[0.00,0.09]$} \\
\hline & $\Sigma_{111}$ & $\operatorname{var}\left(y_{1 i j} \mid \phi_{1 i 1}\right)$ & 57.58 & {$[55.77,59.34]$} \\
\hline & $\Sigma_{121}$ & $\operatorname{cov}\left(y_{1 i j}, y_{2 i j} \mid \phi_{i 1}\right)$ & 35.26 & {$[33.11,37.19]$} \\
\hline & $\Sigma_{221}$ & $\operatorname{var}\left(y_{2 i j} \mid \phi_{2 i 1}\right)$ & 67.56 & {$[65.37,69.63]$} \\
\hline & $\rho_{1}$ & $\operatorname{corr}\left(y_{1 i j}, y_{2 i j} \mid \phi_{i 1}\right)$ & 0.57 & {$[0.55,0.58]$} \\
\hline & $\Lambda_{111}$ & $\operatorname{var}\left(\phi_{1 i 1}\right)$ & 7.95 & {$[5.36,11.31]$} \\
\hline & $\Lambda_{121}$ & $\operatorname{cov}\left(\phi_{1 i 1}, \phi_{2 i 1}\right)$ & 4.58 & {$[2.58,7.14]$} \\
\hline & $\Lambda_{221}$ & $\operatorname{var}\left(\phi_{2 i} \phi_{2 i 1}\right)$ & 5.04 & {$[3.09,7.61]$} \\
\hline \multirow[t]{17}{*}{$2(42 \%)$} & $\beta_{102}^{2.1}$ & Mathematics intercept & 358.04 & {$[357.69,358.41]$} \\
\hline & $\beta_{112}$ & Male & 0.91 & {$[0.71,1.13]$} \\
\hline & $\beta_{122}$ & NHB race & -3.35 & {$[-3.77,-2.96]$} \\
\hline & $\beta_{132}$ & Free or reduced lunch & -2.71 & {$[-3.03,-2.37]$} \\
\hline & $\alpha_{112}$ & Median household income $(\$ 1000)$ & 0.02 & {$[-0.02,0.05]$} \\
\hline & $\beta_{202}$ & Reading intercept & 354.15 & {$[353.77,354.52]$} \\
\hline & $\beta_{212}$ & Male & -0.37 & {$[-0.60,-0.14]$} \\
\hline & $\beta_{222}$ & NHB race & -3.55 & {$[-3.96,-3.09]$} \\
\hline & $\beta_{232}$ & Free or reduced lunch & -2.73 & {$[-3.07,-2.37]$} \\
\hline & $\alpha_{212}$ & Median household income $(\$ 1000)$ & 0.05 & {$[0.02,0.08]$} \\
\hline & $\Sigma_{112}$ & $\operatorname{var}\left(y_{1 i j} \mid \phi_{1 i 2}\right)$ & 36.08 & {$[34.63,37.53]$} \\
\hline & $\Sigma_{122}$ & $\operatorname{cov}\left(y_{1 i j}, y_{2 i j} \mid \phi_{i 2}\right)$ & 21.76 & {$[21.61,22.93]$} \\
\hline & $\Sigma_{222}$ & $\operatorname{var}\left(y_{2 i j} \mid \phi_{2 i 2}\right)$ & 40.52 & {$[38.95,42.17]$} \\
\hline & $\rho_{2}$ & $\operatorname{corr}\left(y_{1 i j}, y_{2 i j} \mid \phi_{i 2}\right)$ & 0.57 & {$[0.56,0.58]$} \\
\hline & $\Lambda_{112}$ & $\operatorname{var}\left(\phi_{1 i 2}\right)$ & 3.35 & {$[2.00,5.20]$} \\
\hline & $\Lambda_{122}$ & $\operatorname{cov}\left(\phi_{1 i 2}, \phi_{2 i 2}\right)$ & 1.65 & {$[0.71,2.84]$} \\
\hline & $\Lambda_{222}$ & $\operatorname{var}\left(\phi_{2 i 2}\right)$ & 1.69 & {$[0.91,2.84]$} \\
\hline \multirow{6}{*}{$\begin{array}{l}\text { Mixing weight } \\
\text { parameters }\end{array}$} & $\gamma_{0}$ & Mixing weight intercept & 0.15 & {$[-0.06,0.37]$} \\
\hline & $\gamma_{1}$ & Male & -0.04 & {$[-0.13,0.04]$} \\
\hline & $\gamma_{2}$ & NHB race & -1.26 & {$[-1.39,-1.11]$} \\
\hline & $\gamma_{3}$ & Free or reduced lunch & -0.89 & {$[-1.01,-0.77]$} \\
\hline & $\delta_{1}$ & Median household income & 0.02 & {$[0.01,0.04]$} \\
\hline & $\tau^{2}$ & $\operatorname{var}\left(\psi_{i}\right)$ & 0.43 & {$[0.25,0.69]$} \\
\hline
\end{tabular}


Table 4 presents the posterior means and 95\% credible intervals $\mathrm{CrI}$ for the model parameters proposed. The results suggest that there are two distinct mixing components, or 'latent subpopulations' of students. Subpopulation 1 contained an estimated $58 \%$ of the overall population and was characterized by comparatively low mean mathematics and reading scores for the reference group $\left(\beta_{101}=349.25\right.$ and $\left.\beta_{201}=344.50\right)$. In addition, NHB race and free- or reduced lunch enrolment were associated with lower mathematics and reading scores. Higher county median income was associated with a slight increase in reading scores $\left(\alpha_{211}=0.05 ; 95 \% \mathrm{CrI}=\right.$ $[0.00,0.09])$. Subpopulation 2 contained the remaining $42 \%$ of the overall population and was associated with roughly a 10-point increase in scores for the reference group compared with subpopulation $1\left(\beta_{102}=358.04\right.$ and $\left.\beta_{202}=354.15\right)$. In both groups, males had lower adjusted reading scores than females (e.g. $\left.\beta_{211}=-1.74 ; 95 \% \mathrm{CrI}=[-1.98,-1.49]\right)$; however, in subpopulation 2, males had higher adjusted mathematics scores $\left(\beta_{112}=0.91 ; 95 \% \mathrm{CrI}=[0.71,1.13]\right)$. These findings are consistent with previous research on gender disparities in standardized test scores, which has shown that boys tend to score lower than girls in reading but modestly higher in mathematics and science, particularly in the upper tails of the test score distribution (Pope and Sydnor, 2010). In terms of variability, subpopulation 1 displayed more individual level and county level heterogeneity than subpopulation 2 . Not surprisingly, the within-subject correlations between mathematics and reading (captured by $\rho_{1}$ and $\rho_{2}$ ) were moderately high for both subpopulations. In addition, the model accounted for almost all of the spatial association in the data: Moran's tests on the county-averaged residuals from the model yielded no evidence of residual spatial correlation ( $p=0.99$ for the mathematics residuals and $p=0.84$ for the reading residuals).

Fig. 7 presents the predicted mathematics and reading scores by county for reference group individuals (i.e. non-Hispanic white females not enrolled in a free- or reduced price lunch programme). Here, the between-county variation reflects both observed differences in county median household income as well as the latent heterogeneity that is captured by the spatial random effects. The spatial patterns of the predicted scores were similar to those for the ordinary least squares residuals in Fig. 2, but with increased smoothing, particularly among the central 'Piedmont' and south-west counties. This feature is expected, since the CAR priors act as spatial smoothers. In general, the north-eastern and central Piedmont counties had higher predicted scores than those in the interior north-east, south central and south-western portions of the state. The predicted mathematics scores for the reference group ranged from 352 to 356 across the state (Fig. 7(a)). Outlined on the map are Union County (along the southern border), which had the highest predicted mathematics score at $356.4(95 \% \mathrm{CrI}=[355.5,357.5])$, and Avery County (in the north-west), which had the lowest predicted score at $352.1(95 \% \mathrm{CrI}=[350.2$, 354.4]). There was also modest variation in the predicted reading scores across the state (Fig. 7(b)). The predicted reading scores ranged from 348 to 353 and showed a spatial pattern similar to the predicted mathematics scores. Camden County, in the north-eastern corner of the state, had the highest predicted reading score $(352.6 ; 95 \% \mathrm{CrI}=[351.0,354.3])$, and Graham County in the south-west had the lowest predicted score $(347.6 ; 95 \% \mathrm{CrI}=[345.2,349.7])$.

Fig. 8 displays four model-estimated bivariate densities for the reference cohort. Fig. 8(a) presents the density for an average county with household income set at the statewide median value and random effects fixed at 0 . Figs 8(b)-8(d) display the densities for three selected counties: Camden County, which had the highest predicted reading score and a top 10\% mathematics score; Bertie County, which was in the bottom 5\% for both mathematics and reading, and Orange County, which had top 5\% predicted mathematics and reading scores. The county-specific densities vary in both their location and their distribution of mass relative to the average density presented in Fig. 8(a). In particular, Camden County shifted towards higher mathematics and 

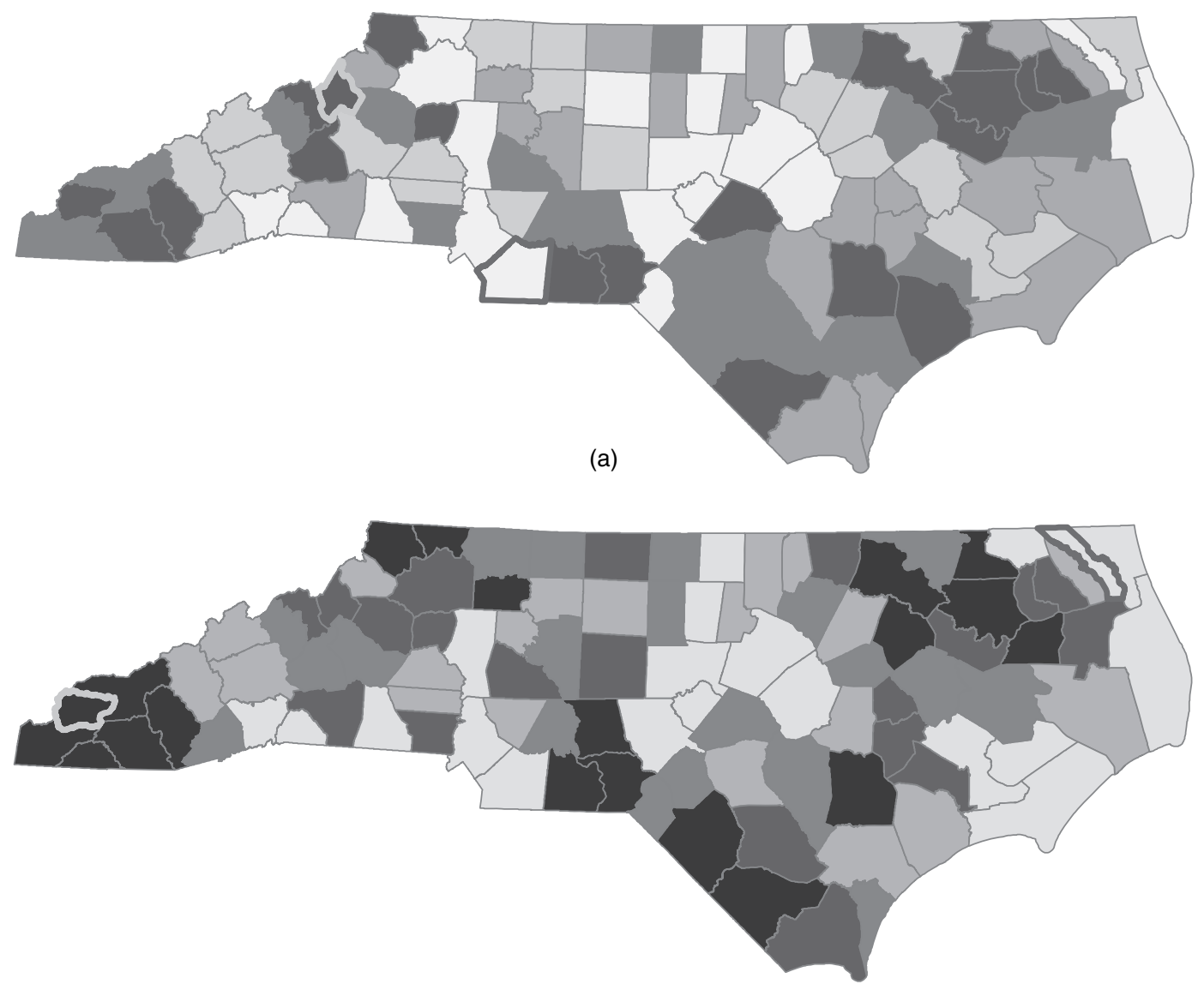

(b)

Fig. 7. Predicted reading scores for reference group, by county: (a) mathematics $(\square, 352.05-353.18$; $\square$, 353.19-353.68; $\square$, 353.69-354.25; $\square$, 354.26-354.91; $\square$, 354.92-356.41; $\square$, highest predicted mathematics score; $\square$, lowest predicted mathematics score); (b) reading ( $\square$, 347.62-349.16; $\square, 349.17-349.45 ; \square$, 349.46-349.93; $\square$, 349.94-350.52; $\square$, 350.53-352.56; $\square$, highest predicted reading score; $\square$, lowest predicted reading score)

reading scores, reflecting the more favourable outcomes for this county, whereas Bertie County had a noticeable shift in mass towards the lower mixture component. Orange County, like Camden County, showed a shift in mass towards more favourable outcomes, but with a longer-tailed distribution, reflecting more heterogeneity for this county.

By integrating across these bivariate densities, we can obtain county-specific predictions of interest. For example, to predict an individual's joint probability of inconsistent or insufficient mastery in both mathematics and reading, defined as a mathematics score of less than 344 and a reading score of less than 342 (Table 1), we evaluated the integral

$$
\left(1-\pi_{i j 2}\right) \int_{-\infty}^{344} \int_{-\infty}^{342} N_{2}\left(\boldsymbol{\eta}_{i j 1}, \boldsymbol{\Sigma}_{1}\right) \mathrm{d} y_{2 i j} \mathrm{~d} y_{1 i j}+\pi_{i j 2} \int_{-\infty}^{344} \int_{-\infty}^{342} N_{2}\left(\boldsymbol{\eta}_{i j 2}, \boldsymbol{\Sigma}_{2}\right) \mathrm{d} y_{2 i j} \mathrm{~d} y_{1 i j} .
$$

A similar approach was used to compute additional joint probabilities of interest. Using the cut-off values that are defined in Table 1, we calculated four joint probabilities of policy interest: 


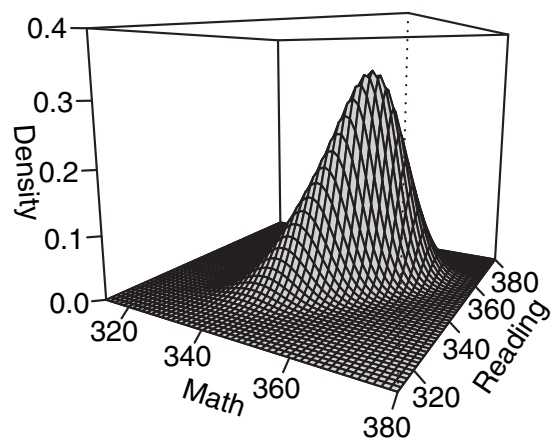

(a)

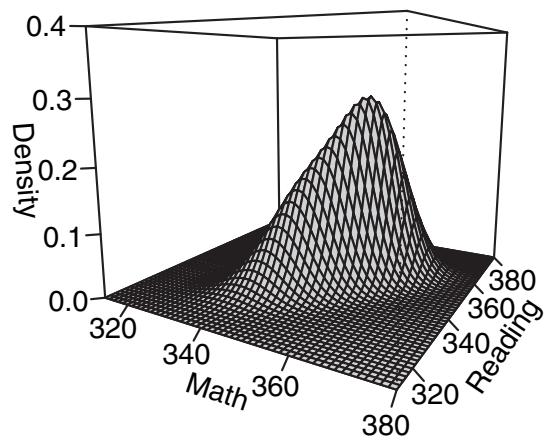

(c)

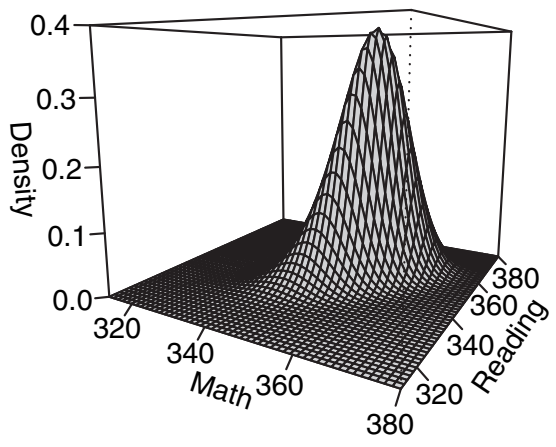

(b)

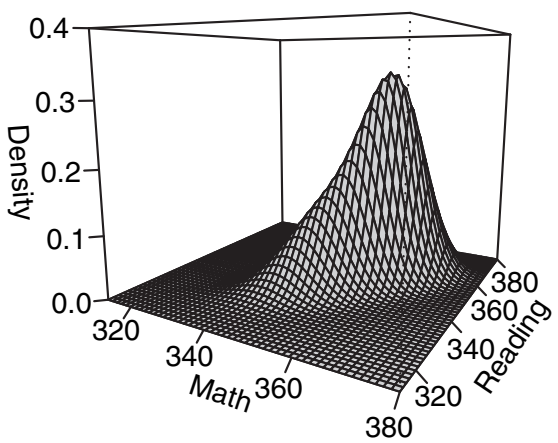

(d)

Fig. 8. Estimated county-specific densities for the reference group: (a) 'average' county; (b) Camden County; (c) Bertie County; (d) Orange County

(a) the probability of inconsistent or insufficient mastery in both mathematics and reading (labelled 'low mathematics-low reading');

(b) the probability of inconsistent or insufficient mathematics, but consistent or superior reading ('low mathematics-high reading');

(c) the probability of consistent or superior mathematics, but inconsistent or insufficient reading ('high mathematics-low reading');

(d) the probability of consistent or superior performance on both examinations ('high mathematics-high reading').

Fig. 9 displays the county-specific averages for these four joint probabilities for the reference group.

In general, the central Piedmont and north-eastern coastal counties had more favourable outcomes than counties in the interior north-east, along the southern border in the east and in the north- and south-west corners of the state. The probability of low mathematics-low reading achievement ranged from 0.04 to 0.13 (Fig. 9(a)), with Camden County having the lowest (0.04; $95 \% \mathrm{CrI}=[0.02,0.06])$ and Bertie County having the highest probability $(0.13 ; 95 \% \mathrm{CrI}=[0.09$, 0.17]). These results are consistent with the predicted densities shown in Fig. 6 for these counties. As Fig. 9(b) indicates, the combination of low mathematics and high reading was a relatively rare event, with average probabilities ranging from 0.02 to 0.07 across the state. This result is not surprising, since students typically perform much better on mathematics than on reading. One 

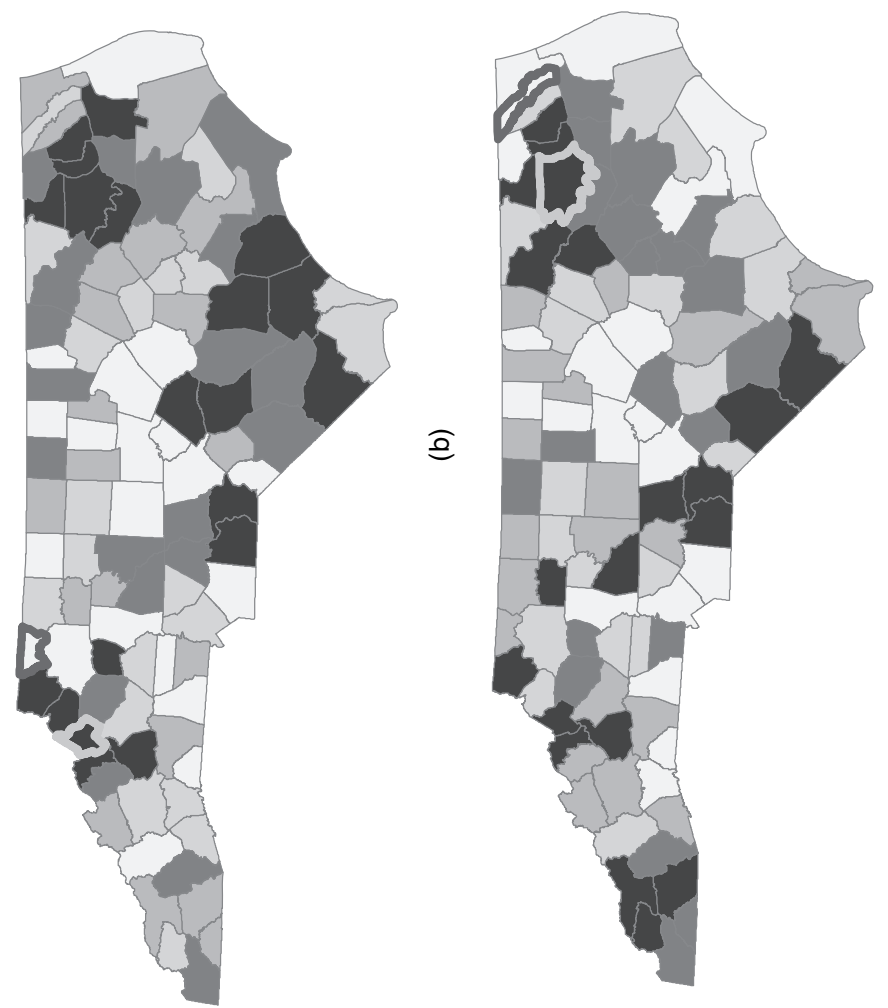

눙무을

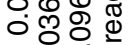

무위등

ले సิष

잉에

110

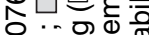

$\circ \bar{m} . \pm=0$

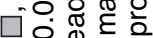

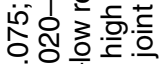

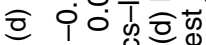

800

$\circ$ 의 등

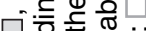

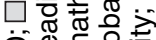

8 은

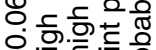

을 을 등응

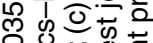

을

등

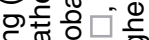

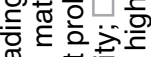

ฮ

으으뭄므.

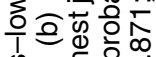

की

을을 중

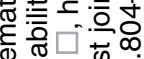

ब

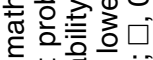

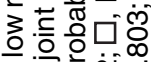
बॅ웡

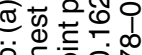
윽등흐오 응 范。

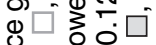

d बै

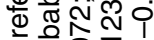

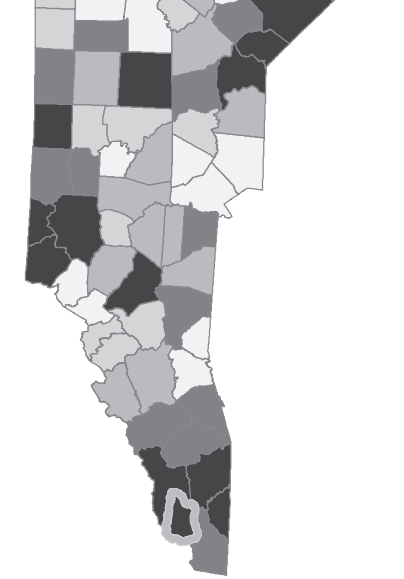

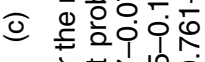

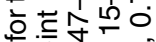

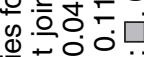

更

言吅造 ه

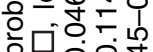
미잉 드워ำ 응ㅇㅇ움ㄷㅁ

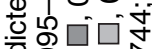

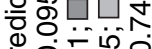
흥뎡ㅇㅇㅇ प0웅

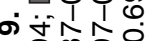

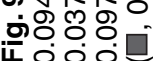


notable exception was Avery County along the central western border, which had the lowest predicted mathematics score (see Fig. 7), but a reading score close to the state average at 349.40 $(95 \% \mathrm{CrI}=[347.67,351.46])$. High mathematics-low reading performance was a more common phenomenon (Fig. 9(c)), ranging from 0.06 to 0.16 state wide, with Graham County having the highest probability $(0.16 ; 95 \% \mathrm{CrI}=[0.11,0.22])$. As Fig. $9(\mathrm{~d})$ suggests, most students in the reference group demonstrated consistent or superior achievement on both examinations. The county averages ranged from 0.69 in Bertie County $(95 \% \mathrm{CrI}=[0.65,0.82])$ to 0.87 in Camden County $(95 \% \mathrm{CrI}=[0.81,0.93])$, again supporting the results that are shown in Figs $6(\mathrm{~b})$ and 6(c).

\section{Discussion}

Closing the achievement gap between high and low performing schools is a necessary step to help all children to succeed in school (Ferguson, 2008). Although the No Child Left Behind Act was designed to help to close the gap by setting standards for evaluating schools, significant disparities remain, both within North Carolina and across the USA (National Center for Education Statistics, 2013). Therefore, it is an important public policy goal to examine regions of varying school performance and to identify factors that are associated with differences in student achievement. This will enable state education officials to direct resources to areas of greatest need. However, because few studies have examined regional differences in student achievement, there remains a need for a systematic approach to identifying geographic areas of high and low performance.

This paper has described a new spatial mixture model designed to meet these goals. The model incorporates individual and region level predictors, accommodates complex dependence structures, enables investigators to examine covariate effects across subgroups of the population and supports region-specific departures from normality through the inclusion of spatial random effects for both the location parameters and the mixing weights. By integrating across this multivariate density, one can obtain region-specific joint, marginal and conditional inferences of interest. In the EOG study, for example, we could compute county-specific joint probabilities of low mathematics-low reading performance, low mathematics, high reading performance, etc. We specified the model within a Bayesian framework and, for posterior computation, we developed a tractable MCMC algorithm that relies in large part on easy-to-sample Gibbs steps.

Our exploratory analysis of the EOG data is, to our knowledge, the first to use advanced multivariate spatial modelling to examine geographic patterns in EOG scores at a refined geographic level. Through our analysis, we found that non-Hispanic black race and enrolment in subsidized lunch programmes were associated with lower test scores. We also found gender gaps in EOG performance, with girls performing substantially better in reading, particularly among students with low EOG scores, and boys scoring slightly higher in mathematics, especially among 'high achieving' students. These results are consistent with previous research on gender disparities in standardized test scores (Pope and Sydnor, 2010; National Center for Education Statistics, 2013), which has shown that boys tend to score lower than girls on standardized reading tests but generally perform better in mathematics and science, particularly in the upper tails of the response distribution (i.e. among high performing students). Together, these findings suggest the need to target two sets of gender disparities: first, in reading among students with low EOG performance and, second, in mathematics among those with high EOG scores. Future work might also examine gender-by-county interactions vis-à-vis a spatial random-coefficients model, to determine whether these disparities vary by region; a finding that has been documented at the national level in prior work (Pope and Sydnor, 2010). 
Our analysis revealed similar spatial patterns for mathematics and reading scores, with the central Piedmont and northern coastal counties displaying higher scores on average than counties in the interior north-east, south central and westernmost portions of the state. These findings could be used to guide county level initiatives to improve elementary education. For example, by identifying counties with comparatively low reading scores, local school boards could introduce school-based literacy programmes to improve vocabulary and reading comprehension. This is especially relevant in counties such as Graham County, which had the highest rate of high mathematics-low reading performance.

Our simulations demonstrated that the mixture model proposed outperforms the conventional, single-component model, even for skewed unimodal data. However, by relying on multivariate normal distributions, we failed to capture fully the skewness that was observed for some of the counties in the second simulation study. An interesting extension, therefore, would be to develop models based on mixtures of skew normal or skew $t$-distributions, building on recent work in the univariate non-mixture setting (Genton and Zhang, 2012; Nathoo and Ghosh, 2013; Zareifard and Khaledi, 2013). This would yield a highly flexible and computationally tractable parametric model that could accommodate both multimodality and extreme skewness.

In our application, we considered two-component mixture models, but higher dimensional mixtures can be envisioned. More generally, one might choose to model the multivariate distribution non-parametrically via infinite mixtures, extending the work of Kottas et al. (2008) to allow for multivariate responses. Extensions to more than two outcomes are also straightforward, although, as the dimension of the problem increases, it may become necessary to impose a structure on the class-specific error covariances $\boldsymbol{\Sigma}_{k}$, to aid in identifying variance components. In high dimensional settings, a factor analytic approach could be used to encourage dimension reduction further.

Our modelling approach should also have broad applicability to other research settings. For example, the spatial mixture model could be applied in reproductive epidemiology to explore joint spatial patterns in birth outcomes and to obtain region-specific joint probabilities of low birth weight and preterm birth. It might also find use in medical imaging as an extension of recent univariate methods (Ismail et al., 2013). In short, the model proposed provides a pragmatic and flexible approach for the joint analysis of multivariate areal data. The MCMC algorithm that was described in Section 3 offers a computationally tractable method for fitting such models.

\section{Acknowledgements}

This work was supported by grant RD-83329301-0 from the US Environmental Protection Agency and was conducted in accordance with a human subjects research protocol approved by Duke University's Institutional Review Board. We are grateful to Claire Osgood for database management and support.

\section{Appendix A: Markov chain Monte Carlo algorithm}

Step 1: update $\gamma_{k}$ and $\delta_{k}$-the full conditional for $p$-dimensional vector $\gamma_{k}(k=2, \ldots, K)$ is given by

$$
\pi\left(\boldsymbol{\gamma}_{k} \mid \cdot\right) \propto \prod_{i=1}^{n} \prod_{j=1}^{n_{i}} \frac{\exp \left(\mathbf{x}_{i j}^{\prime} \boldsymbol{\gamma}_{k}+\mathbf{v}_{i}^{\prime} \boldsymbol{\delta}_{k}+\psi_{i k}\right)}{\sum_{h=1}^{K} \exp \left(\mathbf{x}_{i j}^{\prime} \boldsymbol{\gamma}_{h}+\mathbf{v}_{i}^{\prime} \boldsymbol{\delta}_{h}+\psi_{i h}\right)} N_{p}\left(\boldsymbol{\gamma}_{k} ; \boldsymbol{\mu}_{\gamma}, \boldsymbol{\Sigma}_{\gamma}\right),
$$

where $N_{p}\left(\gamma_{k} ; \cdot\right)$ is a $p$-dimensional normal distribution evaluated at $\gamma_{k}$. Since this full conditional does not have a closed analytic form, we update $\gamma_{k}$ by using a random-walk Metropolis algorithm based on a multivariate $t_{3}\left(s_{g} \mathbf{T}_{k}\right)$ proposal density centred at the previous value, $\gamma_{k}^{\text {old }}$, where the parameter $s_{g}$ 
scales the covariance to achieve optimal acceptance rates, and $\mathbf{T}_{k}$ is a component-specific scale matrix. To improve mixing, we apply the adaptive proposal approach that was developed by Haario et al. (2005), which uses the empirical covariance from an extended burn-in to tune $\mathbf{T}_{k}$. A similar approach can be used to update $\boldsymbol{\delta}_{k}$.

Step 2: update $\psi_{i k}$ - the full conditional for $\psi_{i k}(i=1, \ldots, n ; k=2, \ldots, K)$ is given by

$$
\pi\left(\psi_{i k} \mid \cdot\right) \propto \prod_{j=1}^{n_{i}} \frac{\exp \left(\mathbf{x}_{i j}^{\prime} \gamma_{k}+\mathbf{v}_{i}^{\prime} \boldsymbol{\delta}_{k}+\psi_{i k}\right)}{\sum_{h=1}^{K} \exp \left(\mathbf{x}_{i j}^{\prime} \gamma_{h}+\mathbf{v}_{i}^{\prime} \boldsymbol{\delta}_{h}+\psi_{i h}\right)} N\left(\frac{1}{m_{i}} \sum_{l \in \partial_{i}} \psi_{l k}, \frac{\tau_{k}^{2}}{m_{i}}\right),
$$

where $m_{i}$ and $\partial_{i}$ are defined in equation (4) of Section 3. Since this full conditional does not have a closed form, we update $\psi_{i k}$ by using random-walk Metropolis steps.

Step 3: update $\tau_{k}^{2}$ - assuming an $\operatorname{IG}(g, s)$ prior, draw $\tau_{k}^{2}$ its $\operatorname{IG}\left(g^{*}, s^{*}\right)$ full conditional, where $g^{*}=$ $g+(n-\omega) / 2, s^{*}=s+\boldsymbol{\psi}_{k}^{\prime}(\mathbf{M}-\mathbf{A}) \boldsymbol{\psi}_{k} / 2, n$ is the number of areal units, $\omega=\max (1$, number of 'islands') and $(\mathbf{M}-\mathbf{A})$ is defined in equation (6).

Step 4: update $C_{i j}$-for all $(i, j)$, draw $C_{i j}$ from its full conditional

$$
\pi\left(C_{i j} \mid \cdot\right)=\operatorname{Pr}\left(C_{i j}=k \mid \cdot\right)=\operatorname{Cat}\left(p_{i j k}\right),
$$

where

$$
p_{i j k}=\frac{\pi_{i j k} N_{2}\left(\mathbf{y}_{i j} ; \boldsymbol{\eta}_{i j k}, \boldsymbol{\Sigma}_{k}\right)}{\sum_{h=1}^{K} \pi_{i j h} N_{2}\left(\mathbf{y}_{i j} ; \boldsymbol{\eta}_{i j h}, \boldsymbol{\Sigma}_{h}\right)}
$$

Here, $\operatorname{Cat}\left(p_{i j k}\right)$ denotes a categorical distribution taking the value $k$ with probability $p_{i j k}, \pi_{i j k}$ is the prior probability that $C_{i j}=k$, as given in equation (1) of Section 3 , and $N_{2}\left(\mathbf{y}_{i j} ; \boldsymbol{\eta}_{i j k}, \boldsymbol{\Sigma}_{k}\right)$ denotes the bivariate normal density from equation (1), Section 3 , evaluated at $\mathbf{y}_{i j}$.

Step 5: update $\boldsymbol{\Sigma}_{k}$-assuming an $\operatorname{IW}\left(\kappa_{0}, \mathbf{S}_{0}\right)$ prior, update $\boldsymbol{\Sigma}_{k}$ from its $\operatorname{IW}\left(\kappa^{*}, \mathbf{S}^{*}\right)$ full conditional, where $\kappa^{*}=\kappa_{0}+N_{k}, N_{k}$ is the number of observations in component $k, \mathbf{S}^{*}=\mathbf{S}_{0}+\mathbf{E}_{k}^{\prime} \mathbf{E}_{k}, \mathbf{E}=\mathbf{Y}_{k}-\boldsymbol{\eta}_{k}^{*}$, $\mathbf{Y}_{k}$ is an $N_{k} \times 2$ matrix consisting of $y_{1 i j}$ (the first column) and $y_{2 i j}$ (the second column) response values for all $(i, j)$ in component $k$ and $\boldsymbol{\eta}_{k}^{*}$ is an $N_{k} \times 2$ matrix consisting of $\eta_{1 i j}$ (the first column) and $\eta_{2 i j}$ (the second column) linear predictor values for all $(i, j)$ in component $k$, as defined in equation (1). Use

$$
\boldsymbol{\Sigma}_{k}=\left(\begin{array}{cc}
\sigma_{1 k}^{2} & \rho_{k} \sigma_{1 k} \sigma_{2 k} \\
\rho_{k} \sigma_{1 k} \sigma_{2 k} & \sigma_{2 k}^{2}
\end{array}\right)
$$

to obtain $\sigma_{1 \mid 2, k}^{2}=\left(1-\rho_{k}^{2}\right) \sigma_{1 k}^{2}, \sigma_{2 \mid 1, k}^{2}=\left(1-\rho_{k}^{2}\right) \sigma_{2 k}^{2}, \beta_{1 k}^{*}=\rho_{k} \sigma_{1 k} / \sigma_{2 k}$ and $\beta_{2 k}^{*}=\rho_{k} \sigma_{2 k} / \sigma_{1 k}$.

Step 6: update $\boldsymbol{\beta}_{1 k}$ and $\boldsymbol{\alpha}_{1 k}$-we update $\boldsymbol{\beta}_{1 k}$ and $\boldsymbol{\alpha}_{1 k}$ conditionally on $\mathbf{y}_{2 k}$, where $\mathbf{y}_{2 k}$ denotes the $N_{k} \times 1$ vector of $y_{2 i j}$ response values for all $(i, j)$ in component $k$. Specifically, assuming an $N_{p}\left(\boldsymbol{\mu}_{\beta}, \boldsymbol{\Sigma}_{\beta}\right)$ prior, update $\boldsymbol{\beta}_{1 k}$ from its $N_{p}\left(\mathbf{M}_{\beta_{1 k}}, \mathbf{V}_{\beta_{1 k}}\right)$ full conditional, where

$$
\mathbf{V}_{\beta_{1 k}}=\left\{\boldsymbol{\Sigma}_{\beta}^{-1}+\sigma_{1 \mid 2, k}^{-2}\left(\mathbf{X}_{k}^{\prime} \mathbf{X}_{k}\right)\right\}^{-1}
$$

and

$$
\mathbf{M}_{\beta_{1 k}}=\mathbf{V}_{\beta_{1 k}}\left[\boldsymbol{\Sigma}_{\beta}^{-1} \boldsymbol{\mu}_{\beta}+\sigma_{1 \mid 2, k}^{-2} \mathbf{X}_{k}^{\prime}\left\{\mathbf{y}_{1 k}-\mathbf{V}_{k} \boldsymbol{\alpha}_{1 k}-\boldsymbol{\Phi}_{1 k}-\beta_{1 k}^{*}\left(\mathbf{y}_{2 k}-\boldsymbol{\eta}_{2 k}\right)\right\}\right]
$$

Here, $\mathbf{y}_{1 k}$ denotes the $N_{k} \times 1$ vector of $y_{1 i j}$ response values for all $(i, j)$ in component $k$; $\mathbf{X}_{k}$ denotes the $N_{k} \times p$ individual level design matrix for component $k ; \mathbf{V}_{k}$ is an $N_{k} \times r$ county level design matrix for component $k ; \boldsymbol{\Phi}_{1 k}$ is an $N_{k} \times 1$ stacked vector such that $\phi_{1 i k}$ is replicated for each observation in county $i$ and component $k ; \boldsymbol{\eta}_{2 k}$ is an $N_{k} \times 1$ vector of $\eta_{2 i j}$-values for all $(i, j)$ in component $k$, as defined in equation (1); and $\sigma_{1 \mid 2, k}^{2}$ and $\beta_{1 k}^{*}$ are defined in step 5. A similar set of equations can be used to update the $r \times 1$ vector $\boldsymbol{\alpha}_{1 k}$.

Step 7: update $\boldsymbol{\beta}_{2 k}$ and $\boldsymbol{\alpha}_{2 k}$-we update $\boldsymbol{\beta}_{2 k}$ and $\boldsymbol{\alpha}_{2 k}$ conditionally on $\mathbf{y}_{1 k}$. Specifically, assuming an $N_{p}\left(\boldsymbol{\mu}_{\beta}, \boldsymbol{\Sigma}_{\beta}\right)$ prior, update $\boldsymbol{\beta}_{2 k}$ from its $N_{p}\left(\mathbf{M}_{\beta_{2 k}}, \mathbf{V}_{\beta_{2 k}}\right)$ full conditional, where

$$
\mathbf{V}_{\beta_{2 k}}=\left\{\boldsymbol{\Sigma}_{\beta}^{-1}+\sigma_{2 \mid 1, k}^{-2}\left(\mathbf{X}_{k}^{\prime} \mathbf{X}_{k}\right)\right\}^{-1}
$$

and

$$
\mathbf{M}_{\beta_{2 k}}=\mathbf{V}_{\beta_{2 k}}\left[\boldsymbol{\Sigma}_{\beta}^{-1} \boldsymbol{\mu}_{\beta}+\sigma_{2 \mid 1, k}^{-2} \mathbf{X}_{k}^{\prime}\left\{\mathbf{y}_{2 k}-\mathbf{V}_{k} \boldsymbol{\alpha}_{2 k}-\boldsymbol{\Phi}_{2 k}-\beta_{2 k}^{*}\left(\mathbf{y}_{1 k}-\boldsymbol{\eta}_{1 k}\right)\right\}\right]
$$

Here, $\boldsymbol{\eta}_{1 k}$ is an $N_{k} \times 1$ vector of $\eta_{1 i j}$-values for all $(i, j)$ in component $k$, as defined in equation (1), 
$\sigma_{2 \mid 1, k}^{2}$ and $\beta_{2 k}^{*}$ are defined in step 5, and all other elements are defined in a manner analogous to those in step 6. A similar set of equations can be used to update the $r \times 1$ vector $\boldsymbol{\alpha}_{2 k}$.

Step 8: update $\phi_{i k}$-for $i=1, \ldots, n$, draw the $2 \times 1$ vector $\phi_{i k}$ from its $N_{2}\left(\mathbf{M}_{\phi}, \mathbf{V}_{\phi}\right)$ full conditional, where

$$
\mathbf{V}_{\phi}=\left(N_{i k} \boldsymbol{\Sigma}_{k}^{-1}+m_{i} \boldsymbol{\Lambda}_{k}^{-1}\right)^{-1}
$$

and

$$
\mathbf{M}_{\phi}=\mathbf{V}_{\phi}\left\{\boldsymbol{\Sigma}_{k}^{-1} \mathbf{Z}_{i k}^{\prime}\left(\mathbf{y}_{i k}-\mathbf{X}_{i k} \boldsymbol{\beta}_{k}-\mathbf{V}_{i k} \boldsymbol{\alpha}_{k}\right)+\boldsymbol{\Lambda}_{k}^{-1} \sum_{l \in \partial} \phi_{l k}\right\} .
$$

Here, $N_{i k}$ denotes the component $k$ sample size for county $i ; m_{i}$ is the number of neighbours of county $i$; $\mathbf{Z}_{i k}$ is a $2 N_{i k} \times 2$ matrix with alternating rows of $(1,0)$ and $(0,1) ; \mathbf{y}_{i k}$ is a $2 N_{i k} \times 1$ stacked vector consisting of alternating $Y_{1}$ and $Y_{2}$ response values for each observation in county $i$ and component $k ; \mathbf{X}_{i k}$ is a $2 N_{i k} \times 2 p$ design matrix for county $i$ and component $k$, with rows alternating between $\left(\mathbf{x}_{i j}^{\prime}, \mathbf{0}\right)$ and $\left(\mathbf{0}, \mathbf{x}_{i j}^{\prime}\right)$ for each of the $N_{i k}$ observations in county $i$ and component $k ; \boldsymbol{\beta}_{k}=\left(\boldsymbol{\beta}_{1 k}^{\prime}, \boldsymbol{\beta}_{2 k}^{\prime}\right)^{\prime}$ is a $2 p \times 1$ vector of individual level regression parameters; $\mathbf{V}_{i k}$ is a $2 N_{i k} \times 2 r$ design matrix of county level predictors; and $\boldsymbol{\alpha}_{k}=\left(\boldsymbol{\alpha}_{1 k}^{\prime}, \boldsymbol{\alpha}_{2 k}^{\prime}\right)^{\prime}$ is a corresponding $2 r \times 1$ vector of county level regression coefficients. Step 9: update $\boldsymbol{\Lambda}_{k}$-assuming an $\operatorname{IW}\left(\nu_{0}, \mathbf{D}_{0}\right)$ prior, draw $\boldsymbol{\Lambda}_{k}$ from its $\operatorname{IW}\left(\nu^{*}, \mathbf{D}^{*}\right)$ full conditional, where $\nu^{*}=\nu+n-\omega, n$ is the number of areal units, $\omega$ is defined in step 3 and $\mathbf{D}^{*}=\mathbf{D}_{0}+\boldsymbol{\Phi}_{k}^{*^{\prime}}(\mathbf{M}-\mathbf{A}) \boldsymbol{\Phi}_{k}^{*}$. Here, $\boldsymbol{\Phi}_{k}^{*}$ denotes an $n \times 2$ matrix with first column equal to $\phi_{1 k}=\left(\phi_{11 k}, \ldots, \phi_{1 n k}\right)^{\prime}$ and second column equal to $\phi_{2 k}=\left(\phi_{21 k}, \ldots, \phi_{2 n k}\right)^{\prime}$.

\section{References}

Banerjee, S., Carlin, B. P. and Gelfand, A. E. (2004) Hierarchical Modeling and Analysis for Spatial Data. Boca Raton: Chapman and Hall-CRC.

Besag, J. (1974) Spatial interaction and the statistical analysis of lattice systems (with discussion). J. R. Statist. Soc. B, 36, 192-236.

Besag, J., York, J. and Mollié, A. (1991) Bayesian image restoration, with two applications in spatial statistics. Ann. Inst. Statist. Math., 43, 1-20.

Carlin, B. P. and Banerjee, S. (2002) Hierarchical multivariate CAR models for spatio-temporally correlated survival data (with discussion). Baysn Statist., 7, 45-63.

Celeux, G., Forbes, F., Robert, C. P. and Titterington, D. M. (2006) Deviance information criteria for missing data models. Baysn Anal., 1, 651-674.

Congdon, P. (2010) Random-effects models for migration attractivity and retentivity: a Bayesian methodology. $J$. R. Statist. Soc. A, 173, 755-774.

Ferguson, R. (2008) Toward Excellence with Equity: an Emerging Vision for Closing the Achievement Gap. Cambridge: Harvard Education.

Frühwirth-Schnatter, S. (2006) Finite Mixture and Markov Switching Models. Berlin: Springer.

Gelfand, A. E., Kottas, A. and MacEachern, S. N. (2005) Bayesian nonparametric spatial modeling with Dirichlet process mixing. J. Am. Statist. Ass., 100, 1021-1035.

Gelfand, A. E. and Vounatsou, P. (2003) Proper multivariate conditional autoregressive models for spatial data analysis. Biostatistics, 4, 11-15.

Gelman, A., Carlin, J. B., Stern, H. S. and Rubin, D. B. (2004) Bayesian Data Analysis, 2nd edn. Boca Raton: Chapman and Hall-CRC.

Genton, M. and Zhang, H. (2012) Identifiability problems in some non-Gaussian spatial random fields. Chil. J. Statist., 3, 171-179.

Green, P. J. (1995) Reversible jump Markov chain Monte Carlo computation and Bayesian model determination. Biometrika, 82, 711-732.

Green, P. J. and Richardson, S. (2002) Hidden Markov models and disease mapping. J. Am. Statist. Ass., 97, 1055-1070.

Haario, H., Saksman, E. and Tamminen, J. (2005) Componentwise adaptation for high dimensional MCMC. Computnl Statist., 20, 265-273.

Ismail, S., Sun, W., Nathoo, F. S., Babul, A., Moiseev, A., Beg, M. F. and Virji-Babul, N. (2013) A skew-t spacevarying regression model for the spectral analysis of resting state brain activity. Statist. Meth. Med. Res., 22, $424-438$.

Ji, C., Merl, D., Kepler, T. B. and West, M. (2009) Spatial mixture modelling for unobserved point processes: examples in immunofluorescence histology. Baysn Anal., 4, 297-316.

Jin, X., Carlin, B. P. and Banerjee, S. (2005) Generalized hierarchical multivariate car models for areal data. Biometrics, 61, 950-961. 
Kottas, A., Duan, J. A. and Gelfand, A. E. (2008) Modeling disease incidence data with spatial and spatio-temporal Dirichlet process mixtures. Biometr. J., 50, 29-42.

Kottas, A. and Sansó, B. (2007) Bayesian mixture modeling for spatial Poisson process intensities, with applications to extreme value analysis. J. Statist. Planng Inf., 137, 3151-3163.

Lawson, A. B. and Clark, A. (2002) Spatial mixture relative risk models applied to disease mapping. Statist. Med., 21, 359-370.

Mardia, K. (1988) Multi-dimensional multivariate Gaussian Markov random fields with application to image processing. J. Multiv. Anal., 24, 265-284.

McLachlan, G. and Peel, D. (2000) Finite Mixture Models. New York: Wiley.

Nathoo, F. S. and Ghosh, P. (2013) Skew-elliptical spatial random effect modeling for areal data with application to mapping health utilization rates. Statist. Med., 32, 290-306.

National Center for Education Statistics (2013) The nation's report card: trends in academic progress 2012. Report 2013-456. Institute of Education Sciences, US Department of Education, Washington DC. (Available from http: //nces.ed.gov/nationsreportcard/pubs/main2012/2013456.aspx.)

North Carolina Department of Public Instruction (2006) The North Carolina testing program 2006-2007. North Carolina Department of Public Instruction, Raleigh. (Available from http://www.ncpublicschools . org/docs/accountability/NORTHCgeneralpolicies.pdf.)

North Carolina Department of Public Instruction (2007) Achievement level ranges for the North Carolina end-ofgrade tests: mathematics at grades 3-8. North Carolina Department of Public Instruction, Raleigh. (Available from http://www.ncpublicschools.org/docs/accountability/testing/eog/rangeseog math.pdf.)

North Carolina Department of Public Instruction (2008) Achievement level ranges for the North Carolina end-of-grade tests: reading comprehension at grades 3-8. North Carolina Department of Public Instruction, Raleigh. (Available from http://www.ncpublicschools.org/docs/accountability/testing/ achievelevels/alrangesreading.pdf.)

Pope, D. G. and Sydnor, J. R. (2010) Geographic variation in the gender differences in test scores. J. Econ. Perspect., 24, 95-108.

R Development Core Team (2011) R: a Language and Environment for Statistical Computing. Vienna: R Foundation for Statistical Computing.

Reich, B. J. and Fuentes, M. (2007) A multivariate semiparametric Bayesian spatial modeling framework for hurricane surface wind fields. Ann. Appl. Statist., 1, 249-264.

Richardson, S. (2002) Discussion on 'Bayesian measures of model complexity and fit' (by D. J. Spiegelhalter, N. G. Best, B. P. Carlin and A. van der Linde). J. R. Statist. Soc. B, 64, 626-627.

Sethuraman, J. (1994) A constructive definition of Dirichlet priors. Statist. Sin., 4, 639-650.

Spiegelhalter, D. J., Best, N. G., Carlin, B. P. and van der Linde, A. (2002) Bayesian measures of model complexity and fit (with discussion). J. R. Statist. Soc. B, 64, 583-639.

Stephens, M. (2000) Dealing with label switching in mixture models. J. R. Statist. Soc. B, 62, 795-809.

US Census Bureau (2010) American Community Survey 2005-2009. US Census Bureau, Washington DC. (Available from http://www. census.gov/acs/www/.)

Wall, M. M. and Liu, X. (2009) Spatial latent class analysis model for spatially distributed multivariate binary data. Computnl Statist. Data Anal., 53, 3057-3069.

Zareifard, H. and Khaledi, M. J. (2013) Non-Gaussian modeling of spatial data using scale mixing of a unified skew Gaussian process. J. Multiv. Anal., 114, 16-28.

Zhang, Y., Hodges, J. S. and Banerjee, S. (2009) Smoothed ANOVA with spatial effects as a competitor to MCAR in multivariate spatial smoothing. Ann. Appl. Statist., 3, 1805-1830. 